\title{
Diagnosing CP properties of the 2HDM
}

\section{B. Grzadkowski, ${ }^{a}$ O.M. Ogreid ${ }^{b}$ and P. Osland ${ }^{c}$}

\author{
${ }^{a}$ Faculty of Physics, University of Warsaw, \\ Hoża 69, 00-681 Warsaw, Poland \\ ${ }^{b}$ Bergen University College, \\ Postboks 7030, N-5020 Bergen, Norway \\ ${ }^{c}$ Department of Physics, University of Bergen, \\ Postboks 7803, N-5020 Bergen, Norway \\ E-mail: bohdan.grzadkowski@fuw.edu.pl, omo@hib.no, \\ Per.0sland@ift.uib.no
}

AbStract: We have investigated a Two-Higgs-Doublet Model (2HDM), focusing on CP violation. Various scenarios with spontaneous and explicit breaking of CP have been considered. Some features of CP violation related to a choice of the basis for the two Higgs doublets have been discussed and clarified. Regions in the physical parameter space corresponding to spontaneous and explicit $\mathrm{CP}$ violation have been located and discussed. The possibility to determine parameters of the scalar potential with no reference to Yukawa couplings has been considered and an unavoidable ambiguity has been found. The issue of disentangling spontaneous and explicit CP violation has been investigated.

Keywords: Higgs Physics, Beyond Standard Model, CP violation

ArXiv EPRINT: 1309.6229 


\section{Contents}

1 Introduction $\quad 2$

2 The model 2

3 CP conservation 5

3.1 CPC4: $\lambda_{1}=\lambda_{2}$ and $v_{1}=v_{2} \quad 6$

3.2 CPC5: $\lambda_{1}=\lambda_{2}$ and $\left(\lambda_{1}-\lambda_{3}-\lambda_{4}\right)^{2}=\left|\lambda_{5}\right|^{2} \quad 7$

3.2.1 Case 1: $\lambda_{1}=\lambda_{2}$ and $\lambda_{1}-\lambda_{3}-\lambda_{4}=-\left|\lambda_{5}\right| \quad 7$

3.2.2 Case 2: $\lambda_{1}=\lambda_{2}$ and $\lambda_{1}-\lambda_{3}-\lambda_{4}=+\left|\lambda_{5}\right| \quad 8$

4 CP violation $\quad 9$

4.1 Explicit CP violation 10

$\begin{array}{lll}4.2 & \text { Spontaneous CP violation } & 11\end{array}$

4.2.1 SCPV1: $\operatorname{Im}\left[\left(m_{12}^{2}\right)^{2} \lambda_{5}^{*}\right]=0 \quad 12$

4.2.2 SCPV2: $\lambda_{1}=\lambda_{2}$ and $m_{11}^{2}=m_{22}^{2} \quad 12$

5 Case studies $\quad 13$

$\begin{array}{lll}5.1 & \mathrm{CPC} & 14\end{array}$

$\begin{array}{lll}5.2 & \text { The positivity border } & 15\end{array}$

$\begin{array}{lll}5.3 & \text { The global minimum borders } & 15\end{array}$

$\begin{array}{lll}\text { 5.3.1 SCPV1: } \operatorname{Im}\left[\left(m_{12}^{2}\right)^{2} \lambda_{5}^{*}\right]=0 & 15\end{array}$

5.3.2 SCPV2: $\lambda_{1}=\lambda_{2}$ and $m_{11}^{2}=m_{22}^{2} \quad 16$

$\begin{array}{lll}5.4 & \text { Further illustrations } & 19\end{array}$

6 Disentangling spontaneous and explicit CP-violation $\quad 21$

$\begin{array}{lll}6.1 & \text { Invariants and observables } & 22\end{array}$

6.2 Preliminaries 23

6.3 Determining the potential 24

6.4 An ideal observable? 26

$\begin{array}{llr}7 & \text { Summary } & 26\end{array}$

$\begin{array}{lr}\text { A Minimum conditions } & \mathbf{2 7}\end{array}$

$\begin{array}{lll}\text { A.1 Stationary-point equations for complex vacuum } 28 & 28\end{array}$

B Observable parameters of the potential 28 


\section{Introduction}

We investigate and discuss in detail the issue of $\mathrm{CP}$ violation (CPV) in the scalar sector of the Two-Higgs-Doublet Model (2HDM). In spite of the existing rich literature (see, for example [1]) we believe that it is worth revisiting this issue with particular emphasis on the possibility of spontaneous $\mathrm{CP}$ violation from a phenomenological point of view. The ultimate goal of our study is to identify observables which will distinguish between explicit (ECPV) and spontaneous CP violation (SCPV) without reconstructing the full potential. For early literature on this question, see [2]. The aim of the present paper is more modest: we will determine and display regions of explicit and spontaneous CP violation in the physical parameter space of the model, i.e., in terms of parameters used directly in coupling constants of mass eigenstates, such as mixing angles of neutral scalars, masses, and vacuum expectation values (VEVs).

In general, the parameter regions where spontaneous $\mathrm{CP}$ violation occurs are embedded in regions of explicit CP violation, forming lower-dimensional sub-spaces or manifolds. They can only be located where the potential has two minima of equal depth. However, the converse is not true: not all locations where there are two minima of equal depth correspond to spontaneous CP violation [3]. Thus, if the potential $V$ has two minima labeled $A$ and $B$, spontaneous $\mathrm{CP}$ violation may only occur at the manifolds constituting boundaries between a region where $V_{A}<V_{B}$ and another where $V_{B}<V_{A}$.

We will also discuss the cases of $\mathrm{CP}$ conservation. The trivial ones are at boundaries of the CP-violating parameter space. In addition, we find lower-dimensional manifolds of CP conservation (appearing as points in our two-dimensional plots), totally immersed in a region of explicit $\mathrm{CP}$ violation.

Our discussion is limited to the scalar sector, but is on the other hand rather general in the sense that we do not commit ourselves to any particular scheme for the Yukawa couplings.

The paper is organized as follows. In section 2 we review the minimal model that allows for explicit as well as spontaneous CP violation. In sections 3 and 4 we discuss the conditions for $\mathrm{CP}$ conservation and violation, respectively. In section 5 we illustrate our findings with detailed numerical examples, and in section 6 we discuss the prospects for experimentally establishing CP violation. Section 7 contains a brief summary, one appendix gives explicit minimization conditions, whereas another relates potential parameters to invariants.

\section{The model}

The scalar potential of the $2 \mathrm{HDM}$ shall be parametrized in the standard fashion:

$$
\begin{aligned}
V\left(\Phi_{1}, \Phi_{2}\right)= & -\frac{1}{2}\left\{m_{11}^{2} \Phi_{1}^{\dagger} \Phi_{1}+m_{22}^{2} \Phi_{2}^{\dagger} \Phi_{2}+\left[m_{12}^{2} \Phi_{1}^{\dagger} \Phi_{2}+\text { H.c. }\right]\right\} \\
& +\frac{\lambda_{1}}{2}\left(\Phi_{1}^{\dagger} \Phi_{1}\right)^{2}+\frac{\lambda_{2}}{2}\left(\Phi_{2}^{\dagger} \Phi_{2}\right)^{2}+\lambda_{3}\left(\Phi_{1}^{\dagger} \Phi_{1}\right)\left(\Phi_{2}^{\dagger} \Phi_{2}\right) \\
& +\lambda_{4}\left(\Phi_{1}^{\dagger} \Phi_{2}\right)\left(\Phi_{2}^{\dagger} \Phi_{1}\right)+\frac{1}{2}\left[\lambda_{5}\left(\Phi_{1}^{\dagger} \Phi_{2}\right)^{2}+\text { H.c. }\right] \\
& +\left\{\left[\lambda_{6}\left(\Phi_{1}^{\dagger} \Phi_{1}\right)+\lambda_{7}\left(\Phi_{2}^{\dagger} \Phi_{2}\right)\right]\left(\Phi_{1}^{\dagger} \Phi_{2}\right)+\text { H.c. }\right\},
\end{aligned}
$$


with

$$
\Phi_{i}=\left(\begin{array}{c}
\varphi_{i}^{+} \\
\left(v_{i}+\eta_{i}+i \chi_{i}\right) / \sqrt{2}
\end{array}\right), \quad i=1,2 .
$$

All parameters in (2.1) are real, except for $m_{12}^{2}, \lambda_{5}, \lambda_{6}$ and $\lambda_{7}$, which in general could be complex. In the presence of $\mathrm{CP}$ violation the neutral sector comprises 3 scalars, $H_{i}$ $(i=1,2,3)$, of undefined CP properties, which are defined through the diagonalization of the mass-squared matrix, $\mathcal{M}^{2}$, by an orthogonal rotation matrix $R$ :

$$
\left(\begin{array}{l}
H_{1} \\
H_{2} \\
H_{3}
\end{array}\right)=R\left(\begin{array}{l}
\eta_{1} \\
\eta_{2} \\
\eta_{3}
\end{array}\right)
$$

satisfying

$$
R \mathcal{M}^{2} R^{\mathrm{T}}=\mathcal{M}_{\text {diag }}^{2}=\operatorname{diag}\left(M_{1}^{2}, M_{2}^{2}, M_{3}^{2}\right),
$$

and parametrized e.g. in terms of three rotation angles $\alpha_{i}$ as [4]

$$
R=\left(\begin{array}{ccc}
c_{1} c_{2} & s_{1} c_{2} & s_{2} \\
-\left(c_{1} s_{2} s_{3}+s_{1} c_{3}\right) & c_{1} c_{3}-s_{1} s_{2} s_{3} & c_{2} s_{3} \\
-c_{1} s_{2} c_{3}+s_{1} s_{3} & -\left(c_{1} s_{3}+s_{1} s_{2} c_{3}\right) & c_{2} c_{3}
\end{array}\right)
$$

with $c_{i}=\cos \alpha_{i}, s_{i}=\sin \alpha_{i}$. In eq. (2.3), $\eta_{3} \equiv-\sin \beta \chi_{1}+\cos \beta \chi_{2}$ is the combination of $\chi_{i}$ which is orthogonal to the neutral Nambu-Goldstone boson. Here, $\tan \beta \equiv v_{2} / v_{1}$.

We constrain the model by demanding that there exists a basis for $\left(\Phi_{1}, \Phi_{2}\right)$ in which the VEVs are real and $\lambda_{6}=\lambda_{7}=0$. Then the quartic terms of the potential are invariant under the $\mathcal{Z}_{2}$ symmetry $\Phi_{i} \rightarrow \pm \Phi_{i}$. The symmetry, when imposed upon the whole Lagrangian (except for the soft-breaking quadratic terms in our potential) eliminates flavour-changing neutral currents (FCNC) which otherwise appear in Yukawa interactions. We choose to work in this particular basis. By choosing another basis, we will in general lose its simplicity by introducing non-zero $\lambda_{6}$ and $\lambda_{7}$, and the VEVs may also acquire a phase. This will be illustrated by explicit examples later on. This model is the simplest setting in which the 2HDM may give CP violation.

We shall also ensure vacuum stability, for that we assume that the potential is positive at large field strength irrespective of the direction in the field space. The positivity conditions for the most general case with $\lambda_{6}, \lambda_{7} \neq 0$ (no $\mathcal{Z}_{2}$ symmetry) suitable for a numerical study was formulated in [5], and solved in the geometrical approach of [6]. Here we limit ourselves to the case with $\lambda_{6}=\lambda_{7}=0$, the positivity conditions then read:

$$
\lambda_{1}>0, \quad \lambda_{2}>0, \quad \lambda_{3}+\min \left[0, \lambda_{4}-\left|\lambda_{5}\right|\right]>-\sqrt{\lambda_{1} \lambda_{2}} .
$$

The freedom in choosing a different basis for $\left(\Phi_{1}, \Phi_{2}\right)$ could be parametrized by the following $\mathrm{U}(2)$ transformation: ${ }^{1}$

$$
\left(\begin{array}{c}
\bar{\Phi}_{1} \\
\bar{\Phi}_{2}
\end{array}\right)=e^{i \psi}\left(\begin{array}{cc}
\cos \theta & e^{-i \xi} \sin \theta \\
-e^{i \chi} \sin \theta & e^{i(\chi-\xi)} \cos \theta
\end{array}\right)\left(\begin{array}{l}
\Phi_{1} \\
\Phi_{2}
\end{array}\right) .
$$

\footnotetext{
${ }^{1}$ The parameters of the potential are unaltered by the choice of $\psi$. The transformed VEVs, however, will depend on $\psi$. Thus, a suitable choice of $\psi$ allows us to cancel a common phase of the VEVs.
} 
In our analysis the input parameters will be scalar masses $M_{1,2}, M_{H^{ \pm}}$, the angles $\alpha_{i}$ of the neutral-sector rotation matrix, and

$$
\mu^{2} \equiv \frac{v^{2}}{2 v_{1} v_{2}} \operatorname{Re} m_{12}^{2}
$$

along with a $\mathrm{U}(1)_{\text {em}}$-preserving minimum (defining $\tan \beta$ ) that is taken to be real. Note that reality of the VEVs can always be achieved by an appropriate phase rotation of $\Phi_{i}$ and therefore does not compromise the generality of our approach. It is easy to see that the adopted input parameters are sufficient to determine all the potential parameters. ${ }^{2}$

In our analysis we will assume that the minimum specified by $v_{1,2}$ satifies the constraint $v_{1}^{2}+v_{2}^{2} \sim(246 \mathrm{GeV})^{2}$. However it may happen that this minimum is not the global minimum (vacuum), so we will use the subscript $A$ for our starting minimum to distinguish it from other minima we encounter. Thus,

$$
\left\langle\Phi_{1}\right\rangle_{A}=\frac{1}{\sqrt{2}}\left(\begin{array}{c}
0 \\
v_{1}
\end{array}\right), \quad\left\langle\Phi_{2}\right\rangle_{A}=\frac{1}{\sqrt{2}}\left(\begin{array}{c}
0 \\
v_{2}
\end{array}\right) .
$$

In this paper we are going to study the CP-properties of the model with particular emphasis on distinguishing explicit and spontaneous CP violation. Necessary and sufficient criteria for how to distinguish these two types of $\mathrm{CP}$ violation has been worked out by different groups. In $[8,9]$ a tensorial approach has been used for this purpose, while in $[6,10-12]$ geometric methods have been developed for the same purpose. In our work, we "control" the vacuum since we start with a set of physical masses and the location of the vacuum as input parameters. The parameters of the potential are determined from our set of (physical) input parameters. We have found the approach of $[8,9]$ more convenient for our purposes, and thus we have adopted their tensorial approach. However, we have verified that for the model which was considered in this paper, the conditions for CP conservation obtained in [6, 10-12] coincides with those found in $[8,9]$.

Studying the CP properties of the model, we will sometimes need to express the parameters of the potential also in a different basis. By changing basis, we will in these cases see the true nature of $\mathrm{CP}$ in our model. Any two different bases are related by a U(2)transformation (2.7). In particular, we shall be interested in the cases where a basis exists in which all the parameters of the potential are real $[8,9]$. This is possible for the cases where $\mathrm{CP}$ is conserved or broken spontaneously. We will use a bar-notation to distinguish the parameters of the potential and the fields in this basis, i.e., $\bar{\lambda}_{i}, \bar{m}_{i j}$ and $\bar{\Phi}_{i}$ from the parameters we originally started from.

We shall limit ourselves in this study to a model defined by imposing the $\mathcal{Z}_{2}$ symmetry for dimension- 4 operators in the Lagrangian formulated in a certain initial basis. Then, in this basis, $\lambda_{6}=\lambda_{7}=0$ and tree-level Flavour-Changing Neutral Currents are absent in

\footnotetext{
${ }^{2}$ When $\lambda_{6}=\lambda_{7}=0$, the potential contains 10 real parameters. Two of the mass parameters could be swapped for VEVs via the minimization conditions, see appendix A. The third minimization condition eliminates 1 parameter so that we eventually get 9 parameters. Those could be determined in terms of 3 masses, 3 mixing angles, $\mu^{2}$ and 2 VEVs. For the input masses we use $M_{1}, M_{2}$ and $M_{H^{ \pm}}$, then $M_{3}$ is calculable, see [7] for details. Alternatively, one could take $M_{3}$ as input rather than the ratio $\tan \beta=v_{2} / v_{1}$.
} 
Yukawa couplings [13]. This symmetry will be softly violated by a dimension-2 operator $\Phi_{1}^{\dagger} \Phi_{2}$, here referred to as the $m_{12}^{2}$ term. Note however, that any $\mathrm{U}(2)$ rotation would in general reintroduce non-zero $\lambda_{6}$ and $\lambda_{7}$. In particular, it is worth noticing that a rotation could be adopted to eliminate the $m_{12}^{2}$ term. That would introduce $\lambda_{6}$ - and $\lambda_{7}$-terms, so that the $\mathcal{Z}_{2}$ would appear hardly broken in the other basis. However, the coefficients of those terms would be correlated in such a way that the renormalizability would be preserved exactly in the same manner as in the initial basis containing soft breaking through non-zero $m_{12}^{2}$ with vanishing $\lambda_{6}$ and $\lambda_{7}$.

We shall throughout this paper have repeated need for the phases of $m_{12}^{2}$ and $\lambda_{5}$, so we introduce the following notation for this purpose,

$$
m_{12}^{2}=\left|m_{12}^{2}\right| e^{i \alpha}, \quad \lambda_{5}=\left|\lambda_{5}\right| e^{i \gamma}, \quad 0 \leq \alpha, \gamma<2 \pi .
$$

If $\mathrm{CP}$ is conserved, or spontaneously violated, then a basis exists in which all the parameters of the potential are real. Thus, in this basis the potential (2.1) can be written as

$$
\begin{aligned}
\bar{V}\left(\bar{\Phi}_{1}, \bar{\Phi}_{2}\right)= & -\frac{1}{2}\left\{\bar{m}_{11}^{2} \bar{\Phi}_{1}^{\dagger} \bar{\Phi}_{1}+\bar{m}_{22}^{2} \bar{\Phi}_{2}^{\dagger} \bar{\Phi}_{2}+\bar{m}_{12}^{2}\left[\bar{\Phi}_{1}^{\dagger} \bar{\Phi}_{2}+\text { H.c. }\right]\right\} \\
& +\frac{\bar{\lambda}_{1}}{2}\left(\bar{\Phi}_{1}^{\dagger} \bar{\Phi}_{1}\right)^{2}+\frac{\bar{\lambda}_{2}}{2}\left(\bar{\Phi}_{2}^{\dagger} \bar{\Phi}_{2}\right)^{2}+\bar{\lambda}_{3}\left(\bar{\Phi}_{1}^{\dagger} \bar{\Phi}_{1}\right)\left(\bar{\Phi}_{2}^{\dagger} \bar{\Phi}_{2}\right) \\
& +\bar{\lambda}_{4}\left(\bar{\Phi}_{1}^{\dagger} \bar{\Phi}_{2}\right)\left(\bar{\Phi}_{2}^{\dagger} \bar{\Phi}_{1}\right)+\frac{1}{2} \bar{\lambda}_{5}\left[\left(\bar{\Phi}_{1}^{\dagger} \bar{\Phi}_{2}\right)^{2}+\text { H.c. }\right] \\
& +\left[\bar{\lambda}_{6}\left(\bar{\Phi}_{1}^{\dagger} \bar{\Phi}_{1}\right)+\bar{\lambda}_{7}\left(\bar{\Phi}_{2}^{\dagger} \bar{\Phi}_{2}\right)\right]\left[\left(\bar{\Phi}_{1}^{\dagger} \bar{\Phi}_{2}\right)+\text { H.c. }\right],
\end{aligned}
$$

where now all the $\bar{\lambda}_{i}$ and $\bar{m}_{i j}^{2}$ are real. This basis has the property that if CP is conserved, both VEVs are real, while if $\mathrm{CP}$ is spontaneously violated, the VEV of one doublet is complex. Our starting minimum "A" will in this basis be denoted $\left(\left\langle\bar{\Phi}_{1}\right\rangle_{A},\left\langle\bar{\Phi}_{2}\right\rangle_{A}\right)$.

\section{CP conservation}

In any $2 \mathrm{HDM}, \mathrm{CP}$ is conserved if and only if the three invariants $J_{1}, J_{2}$ and $J_{3}[8,9,15]$ are all real. In a model in which $\lambda_{6}=\lambda_{7}=0$ and the VEVs are real, these invariants can be written in a compact form [14]:

$$
\begin{aligned}
\operatorname{Im} J_{1}= & -\frac{2}{v^{2}} \operatorname{Im}\left[\hat{v}_{\bar{a}}^{*} Y_{a \bar{b}} Z_{b \bar{d}}^{(1)} \hat{v}_{d}\right] \\
= & -\frac{v_{1}^{2} v_{2}^{2}}{v^{4}}\left(\lambda_{1}-\lambda_{2}\right) \operatorname{Im} \lambda_{5} \\
\operatorname{Im} J_{2}= & \frac{2}{v^{4}} \operatorname{Im}\left[\hat{v}_{\bar{b}}^{*} \hat{v}_{\bar{c}}^{*} Y_{b \bar{e}} Y_{c \bar{f}} Z_{e \bar{a} f \bar{d}} \hat{v}_{a} \hat{v}_{d}\right] \\
= & -\frac{v_{1}^{2} v_{2}^{2}}{v^{8}}\left[\left(\left(\lambda_{1}-\lambda_{3}-\lambda_{4}\right)^{2}-\left|\lambda_{5}\right|^{2}\right) v_{1}^{4}+2\left(\lambda_{1}-\lambda_{2}\right) \operatorname{Re} \lambda_{5} v_{1}^{2} v_{2}^{2}\right. \\
& \left.\quad-\left(\left(\lambda_{2}-\lambda_{3}-\lambda_{4}\right)^{2}-\left|\lambda_{5}\right|^{2}\right) v_{2}^{4}\right] \operatorname{Im} \lambda_{5} \\
\operatorname{Im} J_{3}= & \operatorname{Im}\left[\hat{v}_{\bar{b}}^{*} \hat{v}_{\bar{c}}^{*} Z_{b \bar{e}}^{(1)} Z_{c \bar{f}}^{(1)} Z_{e \bar{a} f \bar{d}} \hat{v}_{a} \hat{v}_{d}\right] \\
= & \frac{v_{1}^{2} v_{2}^{2}}{v^{4}}\left(\lambda_{1}-\lambda_{2}\right)\left(\lambda_{1}+\lambda_{2}+2 \lambda_{4}\right) \operatorname{Im} \lambda_{5}
\end{aligned}
$$


The first line of each of these three equations defines the invariant $[8,9]$ (see also $[15,16]$ ), whereas the second line is the model-specific expression for the invariant written out in our starting basis. It is worth noting the absence of $\operatorname{Im} m_{12}^{2}$ above, its presence is hidden since the minimization condition (A.3) has been invoked to express $\operatorname{Im} m_{12}^{2}$ through $\operatorname{Im} \lambda_{5}$.

Thus, CP conservation requires

$$
\operatorname{Im} J_{1}=\operatorname{Im} J_{2}=\operatorname{Im} J_{3}=0 .
$$

The conditions under which $\mathrm{CP}$ is conserved in such a model are described in [14]. They are labeled $\mathrm{CPC} 1$ to $\mathrm{CPC} 5$, and defined by

- CPC1: $v_{1}=0$

- $\mathrm{CPC} 2: v_{2}=0$

- CPC3: $\operatorname{Im} \lambda_{5}=0$

- CPC4: $\lambda_{1}=\lambda_{2}$ and $v_{1}=v_{2}$

- CPC5: $\lambda_{1}=\lambda_{2}$ and $\left(\lambda_{1}-\lambda_{3}-\lambda_{4}\right)^{2}=\left|\lambda_{5}\right|^{2}$

While CPC1-CPC3 are quite trivial it is worth paying some attention to the two remaining conditions. Both require two conditions to be satisfied, and will thus only be satisfied in a lower-dimensional parameter space, as compared with the former three cases.

3.1 CPC4: $\lambda_{1}=\lambda_{2}$ and $v_{1}=v_{2}$

It can be shown that in this case the following $\mathrm{U}(2)$ transformation will make the parameters of the potential and the VEVs simultaneously real:

$$
\left(\begin{array}{c}
\bar{\Phi}_{1} \\
\bar{\Phi}_{2}
\end{array}\right)=e^{i \psi}\left(\begin{array}{cc}
\cos \frac{\pi}{4} & e^{-i \xi} \sin \frac{\pi}{4} \\
i \sin \frac{\pi}{4} & -i e^{-i \xi} \cos \frac{\pi}{4}
\end{array}\right)\left(\begin{array}{c}
\Phi_{1} \\
\Phi_{2}
\end{array}\right)
$$

where $\xi=-\gamma / 2, \psi=-\gamma / 4$ and $\gamma=\arg \left(\lambda_{5}\right)$.

We find that after this transformation

$$
\begin{aligned}
\bar{m}_{12}^{2} & =\left[\operatorname{Re} m_{12}^{2}-2\left|\lambda_{5}\right| v_{1}^{2} \cos ^{2} \frac{\gamma}{2}\right] \sin \frac{\gamma}{2}, \\
\bar{\lambda}_{5} & =-\frac{1}{2}\left(\lambda_{1}-\lambda_{3}-\lambda_{4}+\left|\lambda_{5}\right|\right), \\
\bar{\lambda}_{6} & =0 \\
\bar{\lambda}_{7} & =0 \\
\bar{\lambda}_{1} & =\bar{\lambda}_{2} .
\end{aligned}
$$

Furthermore,

$$
\begin{aligned}
\left\langle\bar{\Phi}_{1}\right\rangle_{A} & =\left(\begin{array}{c}
0 \\
v_{1} \cos \frac{\gamma}{4}
\end{array}\right) \\
\left\langle\bar{\Phi}_{2}\right\rangle_{A} & =\left(\begin{array}{c}
0 \\
v_{1} \sin \frac{\gamma}{4}
\end{array}\right)
\end{aligned}
$$


with

$$
\tan \bar{\beta}=\tan \frac{\gamma}{4}
$$

3.2 CPC5: $\lambda_{1}=\lambda_{2}$ and $\left(\lambda_{1}-\lambda_{3}-\lambda_{4}\right)^{2}=\left|\lambda_{5}\right|^{2}$

Let us consider two different cases for which this can happen:

- Case 1: $\lambda_{1}=\lambda_{2}$ and $\lambda_{1}-\lambda_{3}-\lambda_{4}=-\left|\lambda_{5}\right|$

- Case 2: $\lambda_{1}=\lambda_{2}$ and $\lambda_{1}-\lambda_{3}-\lambda_{4}=+\left|\lambda_{5}\right|$

In both these cases a basis exists in which all the parameters of the potential and the VEVs are simultaneously real.

3.2.1 Case 1: $\lambda_{1}=\lambda_{2}$ and $\lambda_{1}-\lambda_{3}-\lambda_{4}=-\left|\lambda_{5}\right|$

In this case, when $v_{1}+v_{2} \cos (\gamma / 2) \neq 0$ the following $\mathrm{U}(2)$ transformation will make all the parameters of the potential and the VEVs real:

$$
\begin{aligned}
\left(\begin{array}{c}
\bar{\Phi}_{1} \\
\bar{\Phi}_{2}
\end{array}\right)= & \operatorname{sgn}\left(v_{1}+v_{2} \cos \frac{\gamma}{2}\right) \\
& \times e^{i \psi}\left(\begin{array}{c}
\cos \frac{\pi}{4} \\
-\operatorname{sgn}\left(v_{2}-v_{1}\right) e^{i \chi} \sin \frac{\pi}{4} \operatorname{sgn}\left(v_{2}-v_{1}\right) e^{i(\chi-\xi)} \cos \frac{\pi}{4}
\end{array}\right)\left(\begin{array}{l}
\Phi_{1} \\
\Phi_{2}
\end{array}\right)
\end{aligned}
$$

where

$$
\xi=-\frac{\gamma}{2}, \quad \chi=\arctan \frac{2 v_{1} v_{2} \sin \frac{\gamma}{2}}{v_{1}^{2}-v_{2}^{2}}, \quad \psi=-\arctan \frac{v_{2} \sin \frac{\gamma}{2}}{v_{1}+v_{2} \cos \frac{\gamma}{2}}
$$

and $\gamma=\arg \left(\lambda_{5}\right)$.

After this transformation we have

$$
\begin{aligned}
\bar{m}_{12}^{2} & =\frac{\left[\operatorname{Re} m_{12}^{2}-2\left|\lambda_{5}\right| v_{1} v_{2} \cos ^{2} \frac{\gamma}{2}\right] \sqrt{v_{1}^{4}+v_{2}^{4}-2 v_{1}^{2} v_{2}^{2} \cos \gamma}}{2 v_{1} v_{2}} \\
\bar{\lambda}_{5} & =0 \\
\bar{\lambda}_{6} & =0 \\
\bar{\lambda}_{7} & =0 .
\end{aligned}
$$

Furthermore,

$$
\bar{\lambda}_{1}=\bar{\lambda}_{2}
$$

and the transformed minimum becomes

$$
\begin{aligned}
\left\langle\bar{\Phi}_{1}\right\rangle_{A} & =\frac{1}{2}\left(\frac{0}{\sqrt{v_{1}^{2}+v_{2}^{2}+2 v_{1} v_{2} \cos \frac{\gamma}{2}}}\right) \\
\left\langle\bar{\Phi}_{2}\right\rangle_{A} & =\frac{1}{2}\left(\begin{array}{c}
0 \\
\sqrt{v_{1}^{2}+v_{2}^{2}-2 v_{1} v_{2} \cos \frac{\gamma}{2}}
\end{array}\right)
\end{aligned}
$$


meaning the VEVs are all real. This corresponds to CP conservation. However, the value of $\tan \beta$ has also been transformed,

$$
\tan \bar{\beta}=\sqrt{\frac{1+\tan ^{2} \beta-2 \tan \beta \cos \frac{\gamma}{2}}{1+\tan ^{2} \beta+2 \tan \beta \cos \frac{\gamma}{2}}} .
$$

Finally, considering the special case when $v_{1}+v_{2} \cos \frac{\gamma}{2}=0$ (which could occur for $\tan \beta>1$ ), we need to use $\psi=-\frac{\pi}{2}$ in the above $\mathrm{U}(2)$ transformation in order to make the parameters and the VEVs real. The transformed quantities now become

$$
\begin{aligned}
\bar{m}_{12}^{2} & =\frac{\left(\operatorname{Re} m_{12}^{2} v_{2}-2\left|\lambda_{5}\right| v_{1}^{3}\right) \sqrt{\left(v_{2}^{2}+3 v_{1}^{2}\right)\left(v_{2}^{2}-v_{1}^{2}\right)}}{2 v_{1} v_{2}^{2}}, \\
\bar{\lambda}_{5} & =0, \\
\bar{\lambda}_{6} & =0, \\
\bar{\lambda}_{7} & =0,
\end{aligned}
$$

and the transformed minimum is given by

$$
\begin{aligned}
& \left\langle\bar{\Phi}_{1}\right\rangle_{A}=\frac{1}{2}\left(\begin{array}{c}
0 \\
\sqrt{v_{2}^{2}-v_{1}^{2}}
\end{array}\right) \\
& \left\langle\bar{\Phi}_{2}\right\rangle_{A}=\frac{1}{2}\left(\begin{array}{c}
0 \\
\sqrt{v_{2}^{2}+3 v_{1}^{2}}
\end{array}\right)
\end{aligned}
$$

with

$$
\tan \bar{\beta}=\sqrt{\frac{\tan ^{2} \beta+3}{\tan ^{2} \beta-1}} .
$$

3.2.2 Case 2: $\lambda_{1}=\lambda_{2}$ and $\lambda_{1}-\lambda_{3}-\lambda_{4}=+\left|\lambda_{5}\right|$

In this case, when $v_{1}+v_{2} \sin (\gamma / 2) \neq 0$ the following $\mathrm{U}(2)$ transformation will make all the parameters of the potential and the VEVs real:

$$
\begin{aligned}
\left(\begin{array}{c}
\bar{\Phi}_{1} \\
\bar{\Phi}_{2}
\end{array}\right)= & \operatorname{sgn}\left(v_{1}+v_{2} \sin \frac{\gamma}{2}\right) \\
& \times e^{i \psi}\left(\begin{array}{cc}
\cos \frac{\pi}{4} & e^{-i \xi} \sin \frac{\pi}{4} \\
-\operatorname{sgn}\left(v_{2}-v_{1}\right) e^{i \chi} \sin \frac{\pi}{4} \operatorname{sgn}\left(v_{2}-v_{1}\right) e^{i(\chi-\xi)} \cos \frac{\pi}{4}
\end{array}\right)\left(\begin{array}{l}
\Phi_{1} \\
\Phi_{2}
\end{array}\right)
\end{aligned}
$$

where

$$
\xi=\frac{\pi}{2}-\frac{\gamma}{2}, \quad \chi=-\arctan \frac{2 v_{1} v_{2} \cos \frac{\gamma}{2}}{v_{1}^{2}-v_{2}^{2}}, \quad \psi=\arctan \frac{v_{2} \cos \frac{\gamma}{2}}{v_{1}+v_{2} \sin \frac{\gamma}{2}}
$$

and $\gamma=\arg \left(\lambda_{5}\right)$.

We find that after this transformation

$$
\begin{aligned}
\bar{m}_{12}^{2} & =\frac{\left[\operatorname{Re} m_{12}^{2}+2\left|\lambda_{5}\right| v_{1} v_{2} \sin ^{2} \frac{\gamma}{2}\right] \sqrt{v_{1}^{4}+v_{2}^{4}+2 v_{1}^{2} v_{2}^{2} \cos \gamma}}{2 v_{1} v_{2}}, \\
\bar{\lambda}_{5} & =0 \\
\bar{\lambda}_{6} & =0 \\
\bar{\lambda}_{7} & =0
\end{aligned}
$$


Furthermore,

$$
\begin{aligned}
& \left\langle\bar{\Phi}_{1}\right\rangle_{A}=\frac{1}{2}\left(\frac{0}{\sqrt{v_{1}^{2}+v_{2}^{2}+2 v_{1} v_{2} \sin \frac{\gamma}{2}}}\right) \\
& \left\langle\bar{\Phi}_{2}\right\rangle_{A}=\frac{1}{2}\left(\begin{array}{c}
0 \\
\sqrt{v_{1}^{2}+v_{2}^{2}-2 v_{1} v_{2} \sin \frac{\gamma}{2}}
\end{array}\right)
\end{aligned}
$$

meaning they are all real. This corresponds to CP conservation. Furthermore,

$$
\tan \bar{\beta}=\sqrt{\frac{1+\tan ^{2} \beta-2 \tan \beta \sin \frac{\gamma}{2}}{1+\tan ^{2} \beta+2 \tan \beta \sin \frac{\gamma}{2}}} .
$$

Finally, considering the special case when $v_{1}+v_{2} \sin \frac{\gamma}{2}=0$, we have to use $\psi=\frac{\pi}{2}$ in the above $\mathrm{U}(2)$ transformation in order to make the parameters and the VEVs real. The transformed quantities now become

$$
\begin{aligned}
\bar{m}_{12}^{2} & =\frac{\left(\operatorname{Re} m_{12}^{2} v_{2}+2\left|\lambda_{5}\right| v_{1}^{3}\right) \sqrt{\left(v_{2}^{2}+3 v_{1}^{2}\right)\left(v_{2}^{2}-v_{1}^{2}\right)}}{2 v_{1} v_{2}^{2}}, \\
\bar{\lambda}_{5} & =0, \\
\bar{\lambda}_{6} & =0, \\
\bar{\lambda}_{7} & =0,
\end{aligned}
$$

and the transformed minimum is given by

$$
\begin{aligned}
\left\langle\bar{\Phi}_{1}\right\rangle_{A} & =\frac{1}{2}\left(\begin{array}{c}
0 \\
\sqrt{v_{2}^{2}-v_{1}^{2}}
\end{array}\right) \\
\left\langle\bar{\Phi}_{2}\right\rangle_{A} & =\frac{1}{2}\left(\begin{array}{c}
0 \\
\sqrt{v_{2}^{2}+3 v_{1}^{2}}
\end{array}\right)
\end{aligned}
$$

and $\tan \bar{\beta}$ by eq. (3.20).

We note that in both these cases CPC4 and CPC5 (and their subcases), $\bar{\lambda}_{6}$ and $\bar{\lambda}_{7}$ remain zero, but $\tan \beta$ is transformed into a different value $\tan \bar{\beta}$.

\section{CP violation}

In any $2 \mathrm{HDM}, \mathrm{CP}$ is conserved if and only if the three invariants $J_{1}, J_{2}$ and $J_{3}[8,9]$ are all real, see eqs. (3.1)-(3.3).

Thus, $\mathrm{CP}$ violation requires

$$
\operatorname{Im} J_{1} \neq 0 \quad \text { and/or } \quad \operatorname{Im} J_{2} \neq 0 \quad \text { and/or } \quad \operatorname{Im} J_{3} \neq 0 .
$$




\subsection{Explicit CP violation}

According to $[8,9]$, we have to check four invariant quantities, $I_{Y 3 Z}, I_{2 Y 2 Z}, I_{3 Y 3 Z}$ and $I_{6 Z}$ to determine whether $\mathrm{CP}$ is broken spontaneously or explicitly in a CP-violating model. In any $2 \mathrm{HDM}, \mathrm{CP}$ is broken explicitly if at least one of these invariants is non-zero. This means that there exists no basis for which all the parameters of the potential are real.

In a $2 \mathrm{HDM}$ with $\lambda_{6}=\lambda_{7}=0$, and with real VEVs, two of these invariants are zero, and the other two can be written in a compact form:

$$
\begin{aligned}
I_{Y 3 Z}= & \operatorname{Im}\left[Z_{a \bar{c}}^{(1)} Z_{e \bar{b}}^{(1)} Z_{b \bar{e} c \bar{d}} Y_{d \bar{a}}\right] \\
= & 0 \\
I_{2 Y 2 Z}= & \operatorname{Im}\left[Y_{a \bar{b}} Y_{c \bar{d}} Z_{b \bar{a} d \bar{f}} Z_{f \bar{c}}^{(1)}\right] \\
= & \frac{1}{4}\left(\lambda_{1}-\lambda_{2}\right) \operatorname{Im}\left[\left(m_{12}^{2}\right)^{2} \lambda_{5}^{*}\right] \\
= & \frac{v_{1}^{2} v_{2}^{2}}{4 v^{4}}\left(\lambda_{1}-\lambda_{2}\right)\left[4 v^{2} \mu^{2} \operatorname{Re} \lambda_{5}-4 \mu^{4}+v^{4}\left(\operatorname{Im} \lambda_{5}\right)^{2}\right] \operatorname{Im} \lambda_{5}, \\
I_{3 Y 3 Z}= & \operatorname{Im}\left[Z_{a \bar{c} b \bar{d}} Z_{c \bar{e} d \bar{g}} Z_{e \bar{h} f \bar{q}} Y_{g \bar{a}} Y_{h \bar{b}} Y_{q \bar{f}}\right] \\
= & -\frac{1}{8}\left(m_{11}^{2}-m_{22}^{2}\right)\left[\left(\lambda_{1}-\lambda_{3}-\lambda_{4}\right)\left(\lambda_{2}-\lambda_{3}-\lambda_{4}\right)-\left|\lambda_{5}\right|^{2}\right] \operatorname{Im}\left[\left(m_{12}^{2}\right)^{2} \lambda_{5}^{*}\right] \\
= & -\frac{v_{1}^{2} v_{2}^{2}}{8 v^{6}}\left[\left(\lambda_{1}-\lambda_{3}-\lambda_{4}\right)\left(\lambda_{2}-\lambda_{3}-\lambda_{4}\right)-\left|\lambda_{5}\right|^{2}\right] \\
& \times\left[\left(v_{1}^{2}-v_{2}^{2}\right)\left(2 \mu^{2}-v^{2}\left(\lambda_{3}+\lambda_{4}+\operatorname{Re} \lambda_{5}\right)\right)+v^{2}\left(v_{1}^{2} \lambda_{1}-v_{2}^{2} \lambda_{2}\right)\right] \\
& \times\left[4 v^{2} \mu^{2} \operatorname{Re} \lambda_{5}-4 \mu^{4}+v^{4}\left(\operatorname{Im} \lambda_{5}\right)^{2}\right] \operatorname{Im} \lambda_{5}, \\
I_{6 Z}= & \operatorname{Im}\left[Z_{a \bar{b} c \bar{d}} Z_{b \bar{f}}^{(1)} Z_{d \bar{h}}^{(1)} Z_{f \bar{a} j \bar{k}} Z_{k \bar{j} m \bar{n}} Z_{n \bar{m} h \bar{c}}\right] \\
= & 0 .
\end{aligned}
$$

Some comments are here in order:

- The first line of each of these equations is the definition of the invariant $[8,9]$.

- The second line is the model-specific expression of the invariant given in our starting basis before applying the minimization conditions.

- In order to obtain the third form for $I_{2 Y 2 Z}$ we have used the relation (2.8) defining $\mu^{2}$, and (A.3) between $\operatorname{Im} m_{12}^{2}$ and $\operatorname{Im} \lambda_{5}$, obtained by minimization of the potential for real VEVs.

- In order to obtain the third form for $I_{3 Y 3 Z}$ we have expressed $m_{11}^{2}$ and $m_{22}^{2}$ in terms of the $\lambda \mathrm{s}$, according to the minimization conditions (A.1) and (A.2).

In general the $\mathrm{CP}$ violation is explicit if

$$
I_{Y 3 Z} \neq 0 \quad \text { and } / \text { or } \quad I_{2 Y 2 Z} \neq 0 \quad \text { and } / \text { or } \quad I_{3 Y 3 Z} \neq 0 \quad \text { and/or } \quad I_{6 Z} \neq 0 .
$$

However in the simple model defined by eq. (2.1), the non-trivial part of this is

$$
I_{2 Y 2 Z} \neq 0 \quad \text { and } / \text { or } \quad I_{3 Y 3 Z} \neq 0 \text {. }
$$




\subsection{Spontaneous CP violation}

In the case when

$$
I_{Y 3 Z}=I_{2 Y 2 Z}=I_{3 Y 3 Z}=I_{6 Z}=0,
$$

CP is either conserved or broken spontaneously. If, in addition, at least one of the $J_{i}$ is complex, the $\mathrm{CP}$ violation is spontaneous. This means that there exists a choice of basis where all the parameters of the potential are real, but then the vacuum breaks $\mathrm{CP}$ (complex VEVs).

For CP to be broken spontaneously it is necessary that the following five conditions are satisfied simultaneously (failure to do so means the model is CP conserving):

- $v_{1} \neq 0$

- $v_{2} \neq 0$

- $\operatorname{Im} \lambda_{5} \neq 0$

- $\lambda_{1} \neq \lambda_{2}$ or $v_{1} \neq v_{2}$

- $\lambda_{1} \neq \lambda_{2}$ or $\left(\lambda_{1}-\lambda_{3}-\lambda_{4}\right)^{2} \neq\left|\lambda_{5}\right|^{2}$

In addition, one or both of the following conditions emerging from the requirement that $I_{2 Y 2 Z}=0$ and $I_{3 Y 3 Z}=0$ must be satisfied (otherwise the $\mathrm{CP}$ violation would be explicit):

- SCPV1:

$$
\left.4 \frac{\mu^{2}}{v^{2}} \operatorname{Re} \lambda_{5}-4\left(\frac{\mu^{2}}{v^{2}}\right)^{2}+\left(\operatorname{Im} \lambda_{5}\right)^{2}=0 \quad \text { (or equivalently } \quad \operatorname{Im}\left[\left(m_{12}^{2}\right)^{2} \lambda_{5}^{*}\right]=0\right)
$$

- SCPV2:

$$
\lambda_{1}=\lambda_{2}, \quad \lambda_{1}=\lambda_{3}+\lambda_{4}+\operatorname{Re} \lambda_{5}-2 \frac{\mu^{2}}{v^{2}} \quad \text { (or equivalently } \lambda_{1}=\lambda_{2}, m_{11}^{2}=m_{22}^{2} \text { ) }
$$

Note that these conditions refer to the basis defined by eq. (2.1). The above conditions ensure that the potential is indeed CP invariant, and CP is only broken by the VEVs.

An important comment is here in order. Assuming that $\mathrm{U}(1)_{\mathrm{em}}$ is not spontaneously broken, we can, without compromising generality, assume that in any basis $\left\langle\bar{\Phi}_{1}\right\rangle$ is real while $\left\langle\bar{\Phi}_{2}\right\rangle$ is complex. The value of the potential at the minimum will be $V_{\min }=\bar{V}\left(\left\langle\bar{\Phi}_{1}\right\rangle_{A},\left\langle\bar{\Phi}_{2}\right\rangle_{A}\right)$. Complex conjugating both sides of (2.11) it is easy to see that

$$
V_{\min }=\bar{V}\left(\left\langle\bar{\Phi}_{1}\right\rangle_{A},\left\langle\bar{\Phi}_{2}\right\rangle_{A}\right)=\bar{V}\left(\left\langle\bar{\Phi}_{1}\right\rangle_{A},\left\langle\bar{\Phi}_{2}\right\rangle_{A}^{*}\right) .
$$

This means that there exists another minimum of exactly the same depth as our starting minimum A. In the real basis, this second minimum is located at a position in $\Phi_{i \text {-space }}$ that is the complex conjugate of the location of minimum A. Let us label this second minimum B. Thus

$$
\left\langle\bar{\Phi}_{1}\right\rangle_{B}=\left\langle\bar{\Phi}_{1}\right\rangle_{A}, \quad\left\langle\bar{\Phi}_{2}\right\rangle_{B}=\left\langle\bar{\Phi}_{2}\right\rangle_{A}^{*} .
$$

Thus, when we have SCPV, there exist two minima of the same depth which (in the real basis) are complex conjugates of each other. 


\subsubsection{SCPV1: $\operatorname{Im}\left[\left(m_{12}^{2}\right)^{2} \lambda_{5}^{*}\right]=0$}

Invoking the definitions (2.10), the condition $\operatorname{Im}\left[\left(m_{12}^{2}\right)^{2} \lambda_{5}^{*}\right]=0$ becomes:

$$
\left|m_{12}^{2}\right|^{2}\left|\lambda_{5}\right| \operatorname{Im}\left(e^{i(2 \alpha-\gamma)}\right)=0,
$$

which is satisfied when $\sin (2 \alpha-\gamma)=0$. This in turns means that $\cos (2 \alpha-\gamma)= \pm 1$, or

$$
\gamma=2 \alpha+n \pi, \quad n \text { integer } .
$$

In this case, for $\lambda_{6}=\lambda_{7}=0$, the following $\mathrm{U}(2)$ transformation will make all the parameters of the potential real:

$$
\left(\begin{array}{l}
\bar{\Phi}_{1} \\
\bar{\Phi}_{2}
\end{array}\right)=\left(\begin{array}{cc}
1 & 0 \\
0 & e^{i \alpha}
\end{array}\right)\left(\begin{array}{l}
\Phi_{1} \\
\Phi_{2}
\end{array}\right)
$$

This transformation yields

$$
\begin{aligned}
\bar{m}_{12}^{2} & =m_{12}^{2} e^{-i \alpha}=\left|m_{12}^{2}\right|, \\
\bar{\lambda}_{5} & =\lambda_{5} e^{-2 i \alpha}=\left|\lambda_{5}\right| e^{-i(2 \alpha-\gamma)}=\left|\lambda_{5}\right| \cos (2 \alpha-\gamma)= \pm\left|\lambda_{5}\right|, \\
\bar{\lambda}_{6} & =0 \\
\bar{\lambda}_{7} & =0
\end{aligned}
$$

meaning they are all real. This corresponds to spontaneous CP violation. The transformed starting minimum is in this case:

$$
\begin{aligned}
& \left\langle\bar{\Phi}_{1}\right\rangle_{A}=\frac{1}{\sqrt{2}}\left(\begin{array}{c}
0 \\
v_{1}
\end{array}\right) \\
& \left\langle\bar{\Phi}_{2}\right\rangle_{A}=\frac{e^{i \alpha}}{\sqrt{2}}\left(\begin{array}{c}
0 \\
v_{2}
\end{array}\right)
\end{aligned}
$$

\subsubsection{SCPV2: $\lambda_{1}=\lambda_{2}$ and $m_{11}^{2}=m_{22}^{2}$}

In this case, the following $\mathrm{U}(2)$ transformation will make all the parameters of the potential real:

$$
\left(\begin{array}{l}
\bar{\Phi}_{1} \\
\bar{\Phi}_{2}
\end{array}\right)=\left(\begin{array}{cc}
\cos \frac{\pi}{4} & \sin \frac{\pi}{4} \\
-i \sin \frac{\pi}{4} & i \cos \frac{\pi}{4}
\end{array}\right)\left(\begin{array}{c}
\Phi_{1} \\
\Phi_{2}
\end{array}\right)
$$

This transformation yields

$$
\begin{aligned}
\bar{m}_{12}^{2} & =\operatorname{Im}\left(m_{12}^{2}\right), \\
\bar{\lambda}_{5} & =-\frac{1}{4}\left(\lambda_{1}+\lambda_{2}\right)+\frac{1}{2}\left(\lambda_{3}+\lambda_{4}-\operatorname{Re} \lambda_{5}\right), \\
\bar{\lambda}_{6} & =\frac{1}{2} \operatorname{Im} \lambda_{5}, \\
\bar{\lambda}_{7} & =-\frac{1}{2} \operatorname{Im} \lambda_{5},
\end{aligned}
$$


meaning they are all real. This corresponds to spontaneous CP violation. The transformed starting minimum is in this case:

$$
\begin{aligned}
\left\langle\bar{\Phi}_{1}\right\rangle_{A} & =\frac{1}{2}\left(\begin{array}{c}
0 \\
v_{1}+v_{2}
\end{array}\right) \\
\left\langle\bar{\Phi}_{2}\right\rangle_{A} & =\frac{i}{2}\left(\begin{array}{c}
0 \\
v_{2}-v_{1}
\end{array}\right)
\end{aligned}
$$

\section{Case studies}

We will discuss regions in the parameter space of the model limiting ourselves to the following representative cases:

1. $M_{1}=125 \mathrm{GeV}, M_{2}=200 \mathrm{GeV}, M_{H^{ \pm}}=350 \mathrm{GeV}, \mu=250 \mathrm{GeV}, \tan \beta=0.5,1,2$,

2. $M_{1}=125 \mathrm{GeV}, M_{2}=200 \mathrm{GeV}, M_{H^{ \pm}}=350 \mathrm{GeV}, \mu=250 \mathrm{GeV}, \tan \beta=5,10,30$,

3. $M_{1}=125 \mathrm{GeV}, M_{2}=300 \mathrm{GeV}, M_{H^{ \pm}}=500 \mathrm{GeV}, \mu=300 \mathrm{GeV}, \tan \beta=0.5,1,2$,

4. $M_{1}=125 \mathrm{GeV}, M_{2}=300 \mathrm{GeV}, M_{H^{ \pm}}=500 \mathrm{GeV}, \mu=300 \mathrm{GeV}, \tan \beta=5,10,30$.

For these choices we fix $\alpha_{1}$ and search through the $\left(\alpha_{2}, \alpha_{3}\right)$ plane in order to determine regions that are consistent with $\mathrm{CP}$ conservation and/or $\mathrm{CP}$ violation (explicit or spontaneous).

We start with figure 1 where, for $\tan \beta=2$ and $\alpha_{1}= \pm \pi / 6$ it is illustrated how the different constraints reduce the allowed region of the $\left(\alpha_{2}, \alpha_{3}\right)$ parameter space. The rotation angles are defined according to the conventions of [4], so that the allowed ranges are $-\pi / 2<\alpha_{2} \leq \pi / 2$ and $0 \leq \alpha_{3} \leq \pi / 2$. It is worth noticing that for given values of $\tan \beta$ and $\alpha_{1}$, only one of these two quadrants is accessible by allowed models [17]. (At the border, for $\alpha_{2}=0$, we have $M_{3}=M_{2}$.)

The boundaries of the yellow regions will be of particular interest in the following discussion. Green lines and dots indicate locations where CP is conserved. Everywhere else, CP is violated. Red curves and dots indicate where the CP violation is spontaneous.

In the upper panels of figure 1 , the yellow region indicates where a consistent solution for $M_{3}$ (real, and satisfying $M_{3} \geq M_{2}$ ) can be found, otherwise white color is adopted. In the middle panels, positivity (2.6) has been imposed. The pink region indicates where positivity is violated. In the bottom panels, we also impose the constraint that the starting minimum A shall be global. The region forbidden by this constraint is shown in cyan.

As illustrated by the middle and lower panels of figure 1, there are two kinds of borders which are relevant for the model: (i) the border between a region where positivity is satisfied, and where it is not (illustrated by yellow and pink in the middle and bottom panels), and (ii) the border between the region where the starting minimum is the global one, and where it is not (illustrated by yellow and cyan in the bottom panels). We shall refer to these regions as "physical" (yellow), "non-positive" (pink) and "non-global" (cyan).

More results are shown in figures $2-5$. 

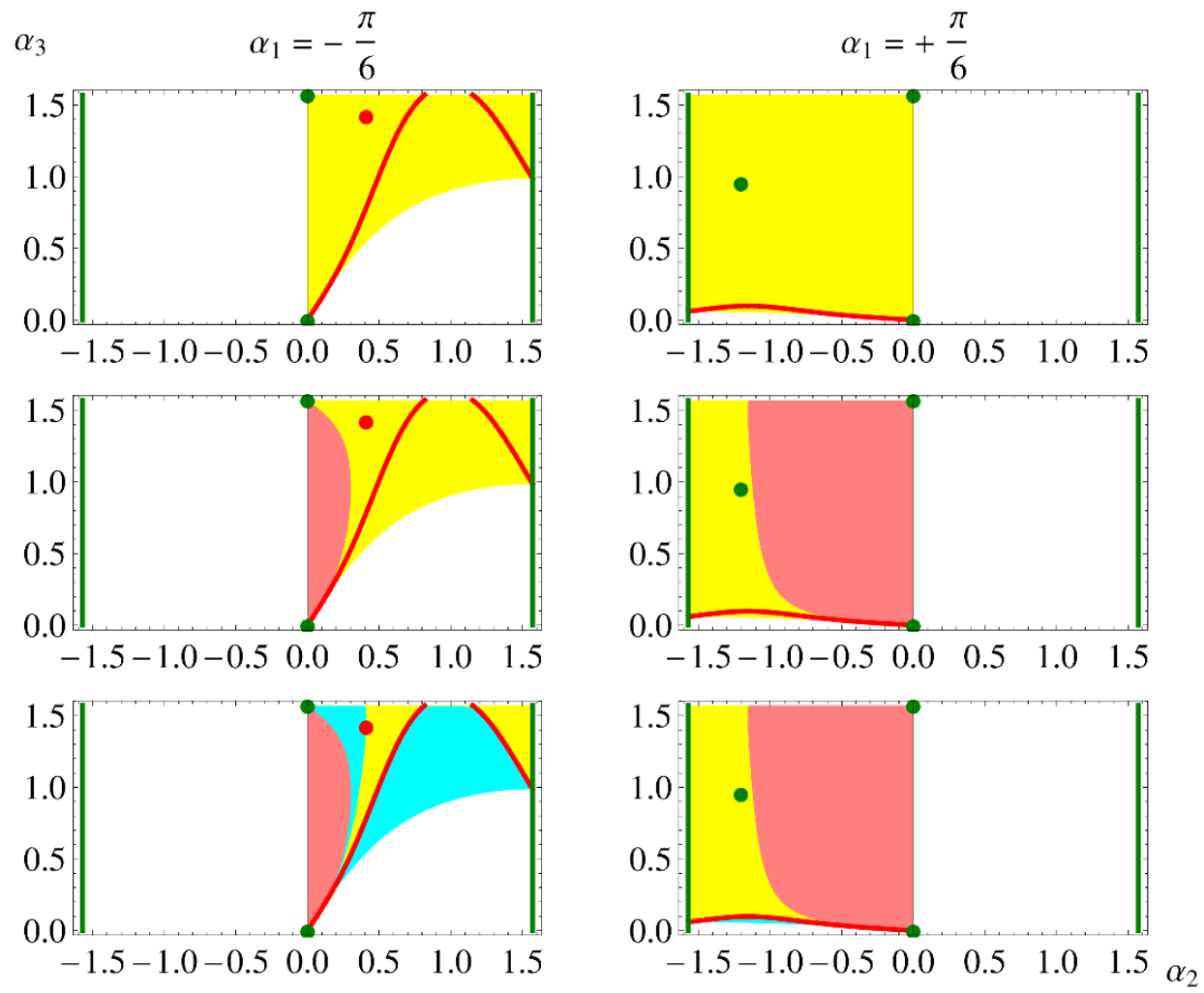

Figure 1. For $\tan \beta=2$, and two values of $\alpha_{1}$ (left: $\alpha_{1}=-\pi / 6$, right: $\alpha_{1}=+\pi / 6$ ), the top panels show the allowed regions (yellow) in the $\alpha_{2}-\alpha_{3}$ space after imposing the constraint $M_{3}>M_{2}$. Red curves correspond to parameters that satisfy the condition (4.9), while red dots satisfy the condition (4.10). Both of these indicate spontaneous CP violation. Green lines and dots indicate locations of $\mathrm{CP}$ conservation. Middle panels: the positivity constraint (2.6) is also imposed (pink region disallowed). Bottom panels: additionally, the global minimum constraint is imposed (cyan region disallowed).

\section{$5.1 \quad$ CPC}

Regions of CPC are denoted by green color, they correspond to parameters for which one of the conditions CPC1-CPC5 specified in section 3 is satisfied. It is worth noting which cases can be realized for our parameter choices. The trivial cases $\mathrm{CPC} 1$ and $\mathrm{CPC} 2$ are not illustrated in our plots. Since we consider only non-degenerate scalar masses, the case CPV3, i.e. $\operatorname{Im} \lambda_{5}=0$ corresponds to [18]:

- $\alpha_{2}= \pm \pi / 2$ (then $R_{11}=R_{12}=R_{23}=R_{33}=0$ and $H_{1}$ is CP odd),

- $\alpha_{2}=0$ and $\alpha_{3}=\pi / 2$ (then $R_{13}=R_{21}=R_{22}=R_{33}=0$ and $H_{2}$ is CP odd),

- $\alpha_{2}=0$ and $\alpha_{3}=0$ (then $R_{13}=R_{23}=R_{31}=R_{32}=0$ and $H_{3}$ is CP odd).

The corresponding regions comprise vertical green lines at the left and right edges of the panels and green dots located in the middle of the lower and upper sides of the panels. For our choices of parameters the case CPC4 is never satisfied. The remaining green 
dots correspond to the case CPC5. It is worth mentioning that these green dots are not isolated points. They just appear as isolated points in our two-dimensional plots. In the full parameter-space, these locations are parts of lower-dimensional manifolds comprising regions of $\mathrm{CP}$ conservation.

\subsection{The positivity border}

As we see from figure 1 there exist two kinds of positivity borders. One can have a non-positive/physical border and a non-positive/non-global border. Along both kinds of borders, the potential will be flat in at least one direction, but bounded from below. When the non-positive/physical border is crossed into the physical region, a global minimum of the potential exists, and is equal to our starting minimum (denoted "A").

When the non-positive/non-global border (left bottom panel in figure 1) is crossed into the non-global region, a global minimum of the potential exists, but our starting minimum A was not the correct one. Another, deeper minimum exists.

\subsection{The global minimum borders}

The region where the starting minimum $A$ is not the global one, is represented in cyan. This region can be adjacent to physical (yellow) regions and to regions where positivity is violated (pink). The former boundaries are manifolds where spontaneous CP violation may occur. In [3], it was shown that the $2 \mathrm{HDM}$ vacuum can be twice degenerate only when a certain symmetry (CP or some other symmetry) of the potential is spontaneously broken. This is consistent with our findings. We discuss these mattes in more detail below.

\subsubsection{SCPV1: $\operatorname{Im}\left[\left(m_{12}^{2}\right)^{2} \lambda_{5}^{*}\right]=0$}

The points satisfying SCPV1 are denoted by red curves. These curves separate a region where the starting minimum (A) is the global minimum (yellow) from a region where it is not. Thus, along the red curves, there are two minima of equal depth. Along the red curves our starting minimum (A) which is real exists alongside another minimum (B) of the same depth (which is complex). The starting minimum can in the basis (2.1)-(2.2) be denoted by

$$
\begin{aligned}
& \left\langle\Phi_{1}\right\rangle_{A}=\frac{1}{\sqrt{2}}\left(\begin{array}{c}
0 \\
v_{1}
\end{array}\right) \\
& \left\langle\Phi_{2}\right\rangle_{A}=\frac{1}{\sqrt{2}}\left(\begin{array}{c}
0 \\
v_{2}
\end{array}\right)
\end{aligned}
$$

which is real. The second minimum which has the same depth is located at

$$
\begin{aligned}
\left\langle\Phi_{1}\right\rangle_{B} & =\frac{1}{\sqrt{2}}\left(\begin{array}{c}
0 \\
v_{1}
\end{array}\right) \\
\left\langle\Phi_{2}\right\rangle_{B} & =\frac{1}{\sqrt{2}}\left(\begin{array}{c}
0 \\
v_{2} e^{-i \gamma}
\end{array}\right)
\end{aligned}
$$


where the $v_{i}$ are the same as for the starting minumum and $\gamma$ is the phase of $\lambda_{5}$, as defined by eq. (2.10).

In the real basis (2.11) we have:

$$
\begin{aligned}
\left\langle\bar{\Phi}_{1}\right\rangle_{A} & =\frac{1}{\sqrt{2}}\left(\begin{array}{c}
0 \\
v_{1}
\end{array}\right) \\
\left\langle\bar{\Phi}_{2}\right\rangle_{A} & =\frac{1}{\sqrt{2}}\left(\begin{array}{c}
0 \\
v_{2} e^{+i \gamma / 2}
\end{array}\right)
\end{aligned}
$$

whereas for the other minumum we get:

$$
\begin{aligned}
\left\langle\bar{\Phi}_{1}\right\rangle_{B} & =\frac{1}{\sqrt{2}}\left(\begin{array}{c}
0 \\
v_{1}
\end{array}\right) \\
\left\langle\bar{\Phi}_{2}\right\rangle_{B} & =\frac{1}{\sqrt{2}}\left(\begin{array}{c}
0 \\
v_{2} e^{-i \gamma / 2}
\end{array}\right)
\end{aligned}
$$

Our potential is CP invariant (as we consider the case of SCPV). Under CP

$$
\Phi_{i} \stackrel{\mathrm{CP}}{\longleftrightarrow} \Phi_{i}^{*}
$$

therefore in particular $V\left(\left\langle\Phi_{1}\right\rangle_{A},\left\langle\Phi_{2}\right\rangle_{A}\right)=V\left(\left\langle\Phi_{1}\right\rangle_{B},\left\langle\Phi_{2}\right\rangle_{B}\right)$. This explains why the curve of SCPV1 separates the forbidden (non-global) and allowed (yellow) regions.

\subsubsection{SCPV2: $\lambda_{1}=\lambda_{2}$ and $m_{11}^{2}=m_{22}^{2}$}

The red dot in figure 1 denotes a point satisfying SCPV2. ${ }^{3}$ This is also on a boundary between a forbidden and an allowed (yellow) region. The cyan region next to the red dot is forbidden because the starting minimum (A) is not the global minimum. Another, deeper minimum with in general complex VEV exists there. In the allowed (yellow) region next to the red dot, the starting minimum (A) is the global minimum. A numerical study shows that for the red dot, the starting minimum (A) which is real exists alongside another minimum (B) which is also real. These have the same depth and are related in the following way:

$$
\begin{aligned}
& \left\langle\Phi_{1}\right\rangle_{B}=\left\langle\Phi_{2}\right\rangle_{A}=\frac{1}{\sqrt{2}}\left(\begin{array}{c}
0 \\
v_{2}
\end{array}\right) \\
& \left\langle\Phi_{2}\right\rangle_{B}=\left\langle\Phi_{1}\right\rangle_{A}=\frac{1}{\sqrt{2}}\left(\begin{array}{c}
0 \\
v_{1}
\end{array}\right) .
\end{aligned}
$$

Clearly, along the border between the allowed (yellow) region and the forbidden region, on the "back", where there is no red curve, there are also two minima of the same depth. However, on this side, as opposed to the "front", where the red curve runs, no

\footnotetext{
${ }^{3}$ These dots are in fact parts of a lower-dimensional manifold of the full parameter space where we have SCPV2. They appear as points only because we show a two-dimensional slice of the full parameter space.
} 
real basis exists, except at one single point, denoted by the red dot where $\mathrm{CP}$ is violated spontaneously. The analytic expression defining the red points is given by eq. (4.10). The values of the VEVs at the red point are specified in eq. (5.4).

Below, we derive analytic expresions that determine the "back border". As a first step, we numerically determined points along the cyan/yellow "back border". Then, after having located these points, the VEVs of both minima were calculated for each of the points. The VEV of the starting minimum (A) was of course the same value that we started out with. The numerical evaluation of the VEV of the second minimum (B) showed that the value of $\left\langle\Phi_{2}\right\rangle_{B}$ is real along the whole "back border". Thus, the VEVs along the border are real for both minima. This simplifies the stationary-point equations a lot, and sets the stage for an analytical study.

Starting with minimum A in which the vacuum is described by our input-parameters $v_{1}$ and $v_{2}$, which we here treat as known quantities, we find the following identities by using the stationary-point equations (A.4)-(A.7):

$$
\begin{aligned}
m_{11}^{2} & =\lambda_{1} v_{1}^{2}+\lambda_{345} v_{2}^{2}-\operatorname{Re}\left(m_{12}^{2}\right) \frac{v_{2}}{v_{1}} \\
m_{22}^{2} & =\lambda_{2} v_{2}^{2}+\lambda_{345} v_{1}^{2}-\operatorname{Re}\left(m_{12}^{2}\right) \frac{v_{1}}{v_{2}} \\
\operatorname{Im}\left(m_{12}^{2}\right) & =\operatorname{Im} \lambda_{5} v_{1} v_{2}
\end{aligned}
$$

Here, we have used the abbreviation $\lambda_{3}+\lambda_{4}+\operatorname{Re} \lambda_{5} \equiv \lambda_{345}$. Using these identities, we arrive at the following expression for the value of the potential at our starting minimum $\mathrm{A}$ :

$$
V\left(\left\langle\Phi_{1}\right\rangle_{A},\left\langle\Phi_{2}\right\rangle_{A}\right)=-\frac{\lambda_{1}}{8} v_{1}^{4}-\frac{\lambda_{2}}{8} v_{2}^{4}-\frac{\lambda_{345}}{4} v_{1}^{2} v_{2}^{2}
$$

Turning now to the second minimum (B) which the numeric study told us was real, we express it as

$$
\begin{aligned}
\left\langle\Phi_{1}\right\rangle_{B} & =\frac{1}{\sqrt{2}}\left(\begin{array}{c}
0 \\
u_{1}
\end{array}\right) \\
\left\langle\Phi_{2}\right\rangle_{B} & =\frac{1}{\sqrt{2}}\left(\begin{array}{c}
0 \\
u_{2}
\end{array}\right)
\end{aligned}
$$

where $u_{1}$ and $u_{2}$ are real, unknown quantities. The minimum $\mathrm{B}$ must also satisfy the stationary-point equations. Thus,

$$
\begin{aligned}
m_{11}^{2} & =\lambda_{1} u_{1}^{2}+\lambda_{345} u_{2}^{2}-\operatorname{Re}\left(m_{12}^{2}\right) \frac{u_{2}}{u_{1}} \\
m_{22}^{2} & =\lambda_{2} u_{2}^{2}+\lambda_{345} u_{1}^{2}-\operatorname{Re}\left(m_{12}^{2}\right) \frac{u_{1}}{u_{2}} \\
\operatorname{Im}\left(m_{12}^{2}\right) & =\operatorname{Im} \lambda_{5} u_{1} u_{2},
\end{aligned}
$$

and the value of the potential at minimum B becomes

$$
V\left(\left\langle\Phi_{1}\right\rangle_{B},\left\langle\Phi_{2}\right\rangle_{B}\right)=-\frac{\lambda_{1}}{8} u_{1}^{4}-\frac{\lambda_{2}}{8} u_{2}^{4}-\frac{\lambda_{345}}{4} u_{1}^{2} u_{2}^{2} .
$$


By combining (5.5) with (5.7) and putting $V\left(\left\langle\Phi_{1}\right\rangle_{A},\left\langle\Phi_{2}\right\rangle_{A}\right)=V\left(\left\langle\Phi_{1}\right\rangle_{B},\left\langle\Phi_{2}\right\rangle_{B}\right)$, we arrive at the following set of equations:

$$
\begin{aligned}
\lambda_{1} v_{1}^{2}+\lambda_{345} v_{2}^{2}-\operatorname{Re}\left(m_{12}^{2}\right) \frac{v_{2}}{v_{1}} & =\lambda_{1} u_{1}^{2}+\lambda_{345} u_{2}^{2}-\operatorname{Re}\left(m_{12}^{2}\right) \frac{u_{2}}{u_{1}} \\
\lambda_{2} v_{2}^{2}+\lambda_{345} v_{1}^{2}-\operatorname{Re}\left(m_{12}^{2}\right) \frac{v_{1}}{v_{2}} & =\lambda_{2} u_{2}^{2}+\lambda_{345} u_{1}^{2}-\operatorname{Re}\left(m_{12}^{2}\right) \frac{u_{1}}{u_{2}} \\
v_{1} v_{2} & =u_{1} u_{2} \\
\frac{\lambda_{1}}{8} v_{1}^{4}+\frac{\lambda_{2}}{8} v_{2}^{4}+\frac{\lambda_{345}}{4} v_{1}^{2} v_{2}^{2} & =\frac{\lambda_{1}}{8} u_{1}^{4}+\frac{\lambda_{2}}{8} u_{2}^{4}+\frac{\lambda_{345}}{4} u_{1}^{2} u_{2}^{2}
\end{aligned}
$$

This is a set of four equations with only two unknown $\left(u_{1}\right.$ and $\left.u_{2}\right)$. Combining (5.11) and (5.12) we solve for $u_{1}$ and $u_{2}$ (picking the only real, positive solution not corresponding to minimum $A$ ) to get

$$
u_{1}=\sqrt[4]{\frac{\lambda_{2}}{\lambda_{1}}} v_{2}, \quad u_{2}=\sqrt[4]{\frac{\lambda_{1}}{\lambda_{2}}} v_{1}
$$

Thus, we have found that the VEVs of the second minimum (B) along the "back border" are given by

$$
\begin{aligned}
& \left\langle\Phi_{1}\right\rangle_{B}=\frac{1}{\sqrt{2}}\left(\begin{array}{c}
0 \\
\sqrt[4]{\frac{\lambda_{2}}{\lambda_{1}}} v_{2}
\end{array}\right), \\
& \left\langle\Phi_{2}\right\rangle_{B}=\frac{1}{\sqrt{2}}\left(\begin{array}{c}
0 \\
\sqrt[4]{\frac{\lambda_{1}}{\lambda_{2}}} v_{1}
\end{array}\right) .
\end{aligned}
$$

We see that this simplifies to the VEVs we found for minimum B in the case of SCPV2, see eq. (5.4). Inserting these VEVs into either (5.9) or (5.10), we arrive at the same equation:

$$
\operatorname{Re}\left(m_{12}^{2}\right)=\left(\lambda_{345}-\sqrt{\lambda_{1}} \sqrt{\lambda_{2}}\right) v_{1} v_{2}
$$

This turns out to be the equation defining the curve that constitutes the "back border". However, this curve can be expressed in many different ways by using the equations in (5.5) to rewrite it. After some algebra, we find that (5.14) implies

$$
\sqrt{\lambda_{1}} m_{22}^{2}-\sqrt{\lambda_{2}} m_{11}^{2}=0
$$

This expression does not explicitly contain $v_{1}$ or $v_{2}$, and clearly shows that whenever we have $\operatorname{SCPV} 2\left(\lambda_{1}=\lambda_{2}, m_{11}^{2}=m_{22}^{2}\right)$, this equation is satisfied by default.

We note that whenever (5.15) is satisfied, the potential is invariant under the following transformation:

$$
\begin{aligned}
\Phi_{1} & \rightarrow \sqrt[4]{\frac{\lambda_{2}}{\lambda_{1}}} \Phi_{2}^{*} \\
\Phi_{2} & \rightarrow \sqrt[4]{\frac{\lambda_{1}}{\lambda_{2}}} \Phi_{1}^{*}
\end{aligned}
$$

Thus, we have identified an additional discrete symmetry of the potential along the "back border" that explains why we have two minima of equal depth along the curve defined by (5.15). In fact, we easily see that the two minima $A$ and $B$ transform into each other under this transformation. 

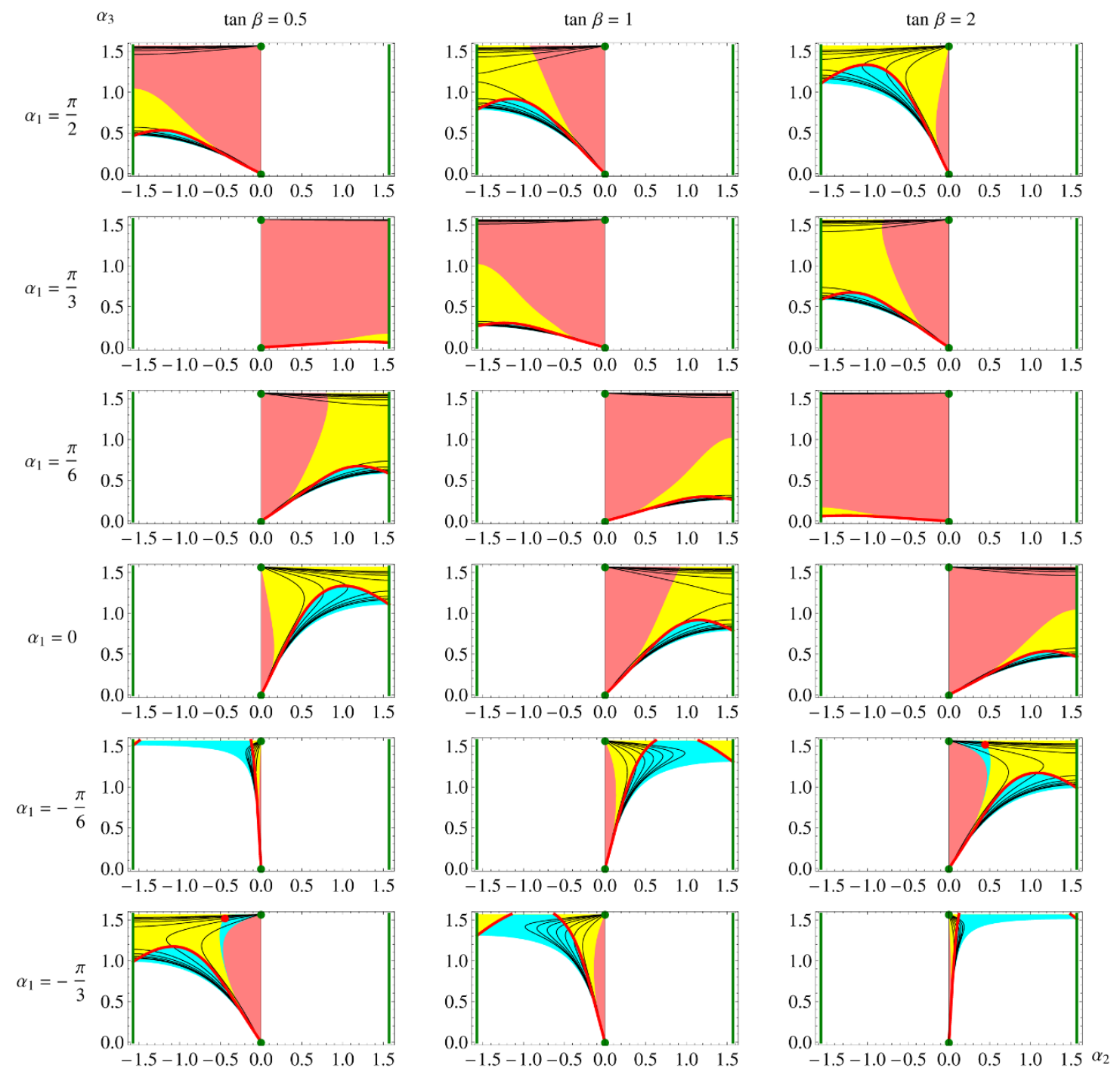

Figure 2. Similar to the bottom panels of figure 1, for $M_{1}=125 \mathrm{GeV}, M_{2}=200 \mathrm{GeV}, M_{H^{ \pm}}=$ $350 \mathrm{GeV}, \mu=250 \mathrm{GeV}$, three values of $\tan \beta$ (left to right: $0.5,1,2)$ and six values of $\alpha_{1}$ (top to bottom: $\pi / 2, \pi / 3, \pi / 6,0,-\pi / 6,-\pi / 3)$. White: excluded because $M_{3}^{2}<M_{2}^{2}$; Pink: excluded by non-positivity; Cyan: excluded by the global minimum constraint. The solid black contours indicate constant values of $M_{3}=300,400, \cdots \mathrm{GeV}$, the curves are moving outwards from the vertical line $\alpha_{2}=0$ as $M_{3}$ increases.

\subsection{Further illustrations}

In figures $2-5$ we illustrate regions of ECPV and SCPV for the parameter choices specified at the beginning of section 5 .

The following symmetry (discussed in section 3.1 of [5]) can be observed in figure 2 and figure 4:

$$
\tan \beta \leftrightarrow \cot \beta, \quad \alpha_{1} \leftrightarrow \frac{1}{2} \pi-\alpha_{1}, \quad \alpha_{2} \leftrightarrow-\alpha_{2} .
$$

The cases shown in figure 1 correspond to the third and fifth row in the right-hand column of figure 4 . 

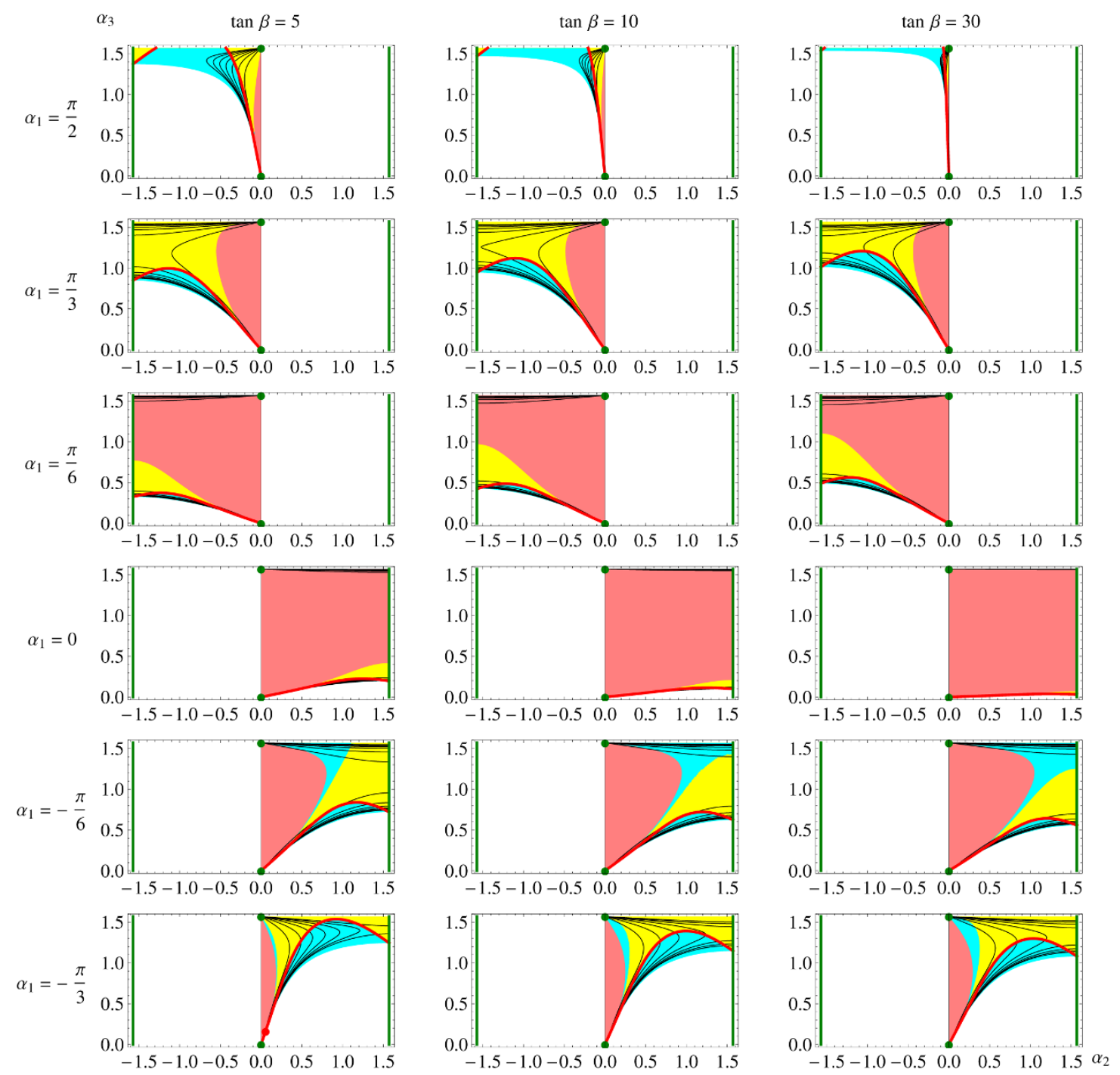

Figure 3. Similar to figure 2 for $\tan \beta=5,10$ and 30 .

In all panels of figures 2-5 positivity and the global-minimum constraint are imposed. We note that only one of the cases studied in figure 1 exhibits SCPV2. The red dot (SCPV2) appears at values of $\alpha_{1}$ between -0.83 and -0.47 . The red dot then appears around $(0, \pi / 2)$, moves somewhat down and to the right and then up again to disappear at $(0.49, \pi / 2)$ as $\alpha_{1}$ varies in this interval.

In some of these panels (for low $\tan \beta$ ), we note the appearance of a green dot, indicating $\mathrm{CP}$ conservation inside a yellow region (recall that the yellow region denotes explicit $\mathrm{CP}$ violation). As already mentioned, the dot corresponds to the case CPC5.

In our examples, the high- $\tan \beta$ cases (figures 3 and 5 ) do not exhibit any of the isolated points (SCPV2) of spontaneous CP violation, only SCPV1 (red border between the blue and yellow regions) and explicit CP violation (yellow). Furthermore, there is no isolated point of $\mathrm{CP}$ conservation inside the yellow regions either. In this sense, the low-tan $\beta$ cases have more structure. 
$\alpha_{3}$
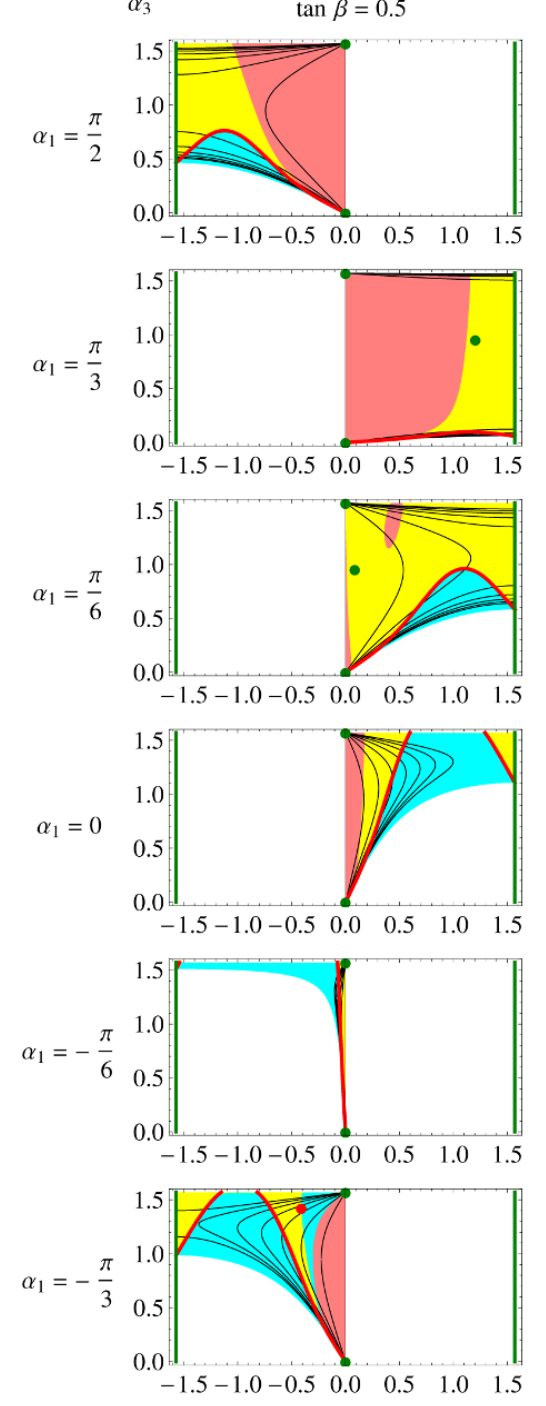

$\tan \beta=1$
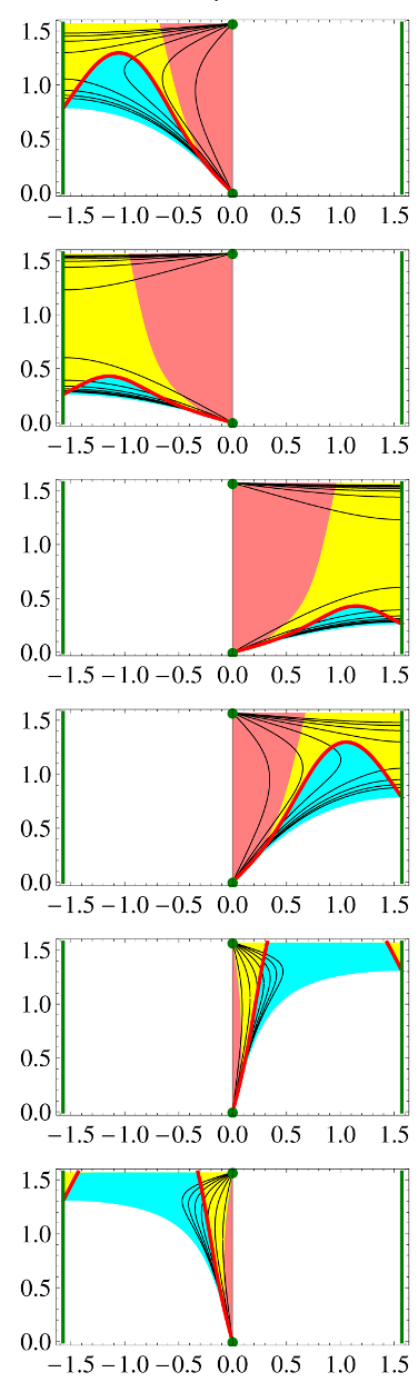

$\tan \beta=2$
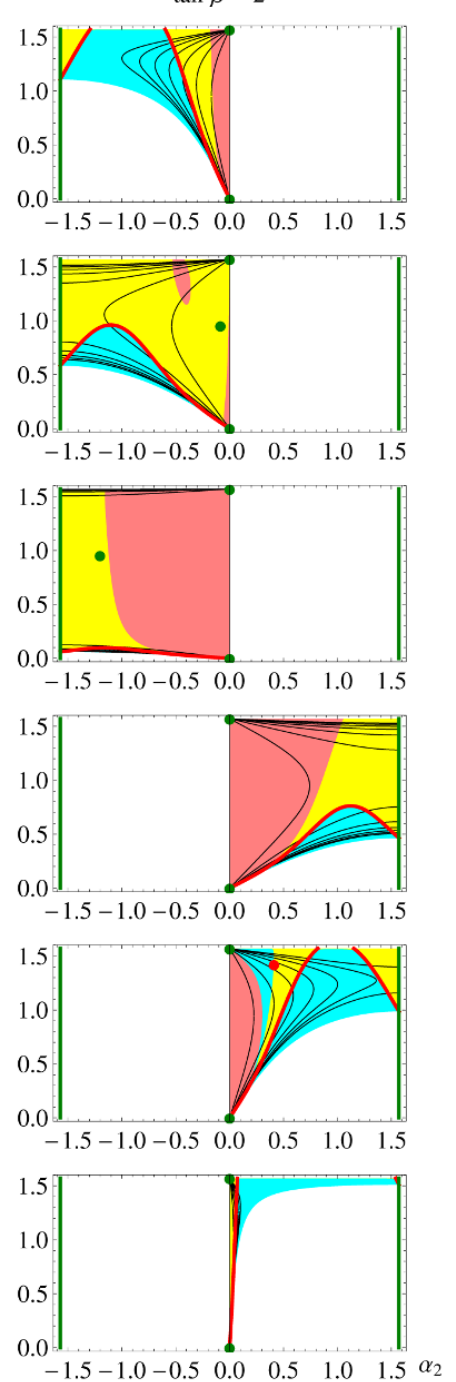

Figure 4. Similar to the bottom panels of figure 1, for $M_{1}=125 \mathrm{GeV}, M_{2}=300 \mathrm{GeV}, M_{H^{ \pm}}=$ $500 \mathrm{GeV}, \mu=300 \mathrm{GeV}$, three values of $\tan \beta$ (left to right: $0.5,1,2)$ and six values of $\alpha_{1}$ (top to bottom: $\pi / 2, \pi / 3, \pi / 6,0,-\pi / 6,-\pi / 3)$. White: excluded because $M_{3}^{2}<M_{2}^{2}$; Pink: excluded by non-positivity; Cyan: excluded by the global minimum constraint. The solid black contours indicate constant values of $M_{3}=400,500, \cdots \mathrm{GeV}$, the curves are moving outwards from the vertical line $\alpha_{2}=0$ as $M_{3}$ increases.

In order to focus on $\mathrm{CP}$ violation, we have not exhibited the impact of other constraints. When these are imposed, significant parts of the remaining (yellow) parameter space are excluded. For the case of Type II Yukawa couplings, see for example [20, 21].

\section{Disentangling spontaneous and explicit CP-violation}

The ultimate goal of this study should be to propose a phenomenological strategy that allows one to disentangle spontaneous from explicit CP-violation. There are several comments in order, regarding that goal. 

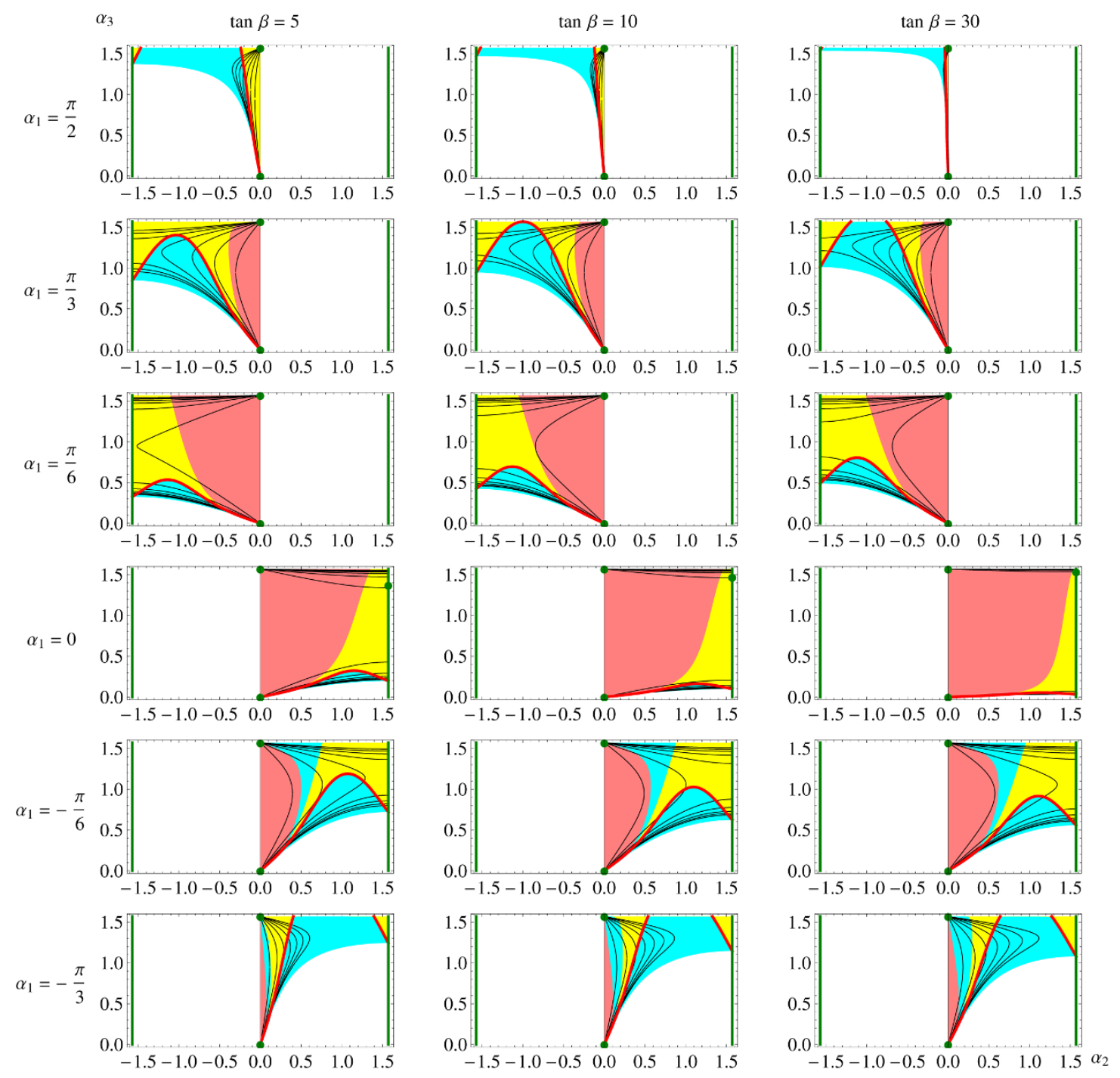

Figure 5. Similar to figure 4 for $\tan \beta=5,10$ and 30 .

\subsection{Invariants and observables}

Any physical observable quantity must be independent of our choice of basis. This is the motivation behind giving a basis-independent formulation of the 2HDM. When we study a particular model and choose a particular basis suitable for the study, this amounts to assigning values to certain parameters, or constraining them by assuming relations between the parameters of the model.

When we write out the full algebraic expression for an invariant quantity in the completely general $2 \mathrm{HDM}$, without choosing any particular basis, we get an expression that is itself manifestly invariant. By this, we mean that applying the transformation rules for each parameter in the expression under a change of basis, we get exactly the same algebraic expression in terms of the transformed parameters. 
When we write out the algebraic expressions for invariant quantities in a 2HDM where we have chosen a particular basis, the resulting algebraic expressions are not always manifestly invariant anymore. So if we now apply the transformation rules for each parameter in the expression under a change of basis, we may get a different algebraic expression in terms of the transformed parameters.

When we perform a measurement, we determine a quantity that is basis independent. However, in a $2 \mathrm{HDM}$ where a particular basis has been chosen, this measurement will correspond to a basis-specific algebraic expression (that is not necessarily invariant) for the measured invariant. In this sense we may say that we interpret the measured invariant quantity as corresponding to the non-invariant basis-specific algebraic expression in our model. ${ }^{4}$

In our model, even without specifying the Yukawa sector, experiments will let us measure certain combinations of parameters. By combining measurements, we may thus determine parameters of our model. However, a parameter can only be determined from experiments if there exists an invariant (or function of invariants) that in the model simplifies to this parameter. Examples of observable parameters that can be determined uniquely in our model are $\lambda_{3}$ and $\lambda_{4}$, while there is a twofold ambiguity that prevents us from determining $\lambda_{1}$ and $\lambda_{2}$ uniquely. This twofold ambiguity is discussed in detail in appendix B.

In that appendix, we arrive at the following list of nine independent observables:

$$
\left(v_{1}^{2}+v_{2}^{2}\right),\left(v_{1}^{2}-v_{2}^{2}\right)^{2},\left(\lambda_{1}+\lambda_{2}\right),\left(\lambda_{1}-\lambda_{2}\right)^{2}, \lambda_{3}, \lambda_{4}, \operatorname{Re} \lambda_{5},\left(\operatorname{Im} \lambda_{5}\right)^{2}, \mu^{2},
$$

meaning that we can determine all the parameters of the potential, except for those that would let us distinguish one doublet from the other. Of course, specifying the Yukawa sector would normally allow us to resolve the ambiguities.

Since the conditions for spontaneous CP violation are symmetric under an exchange of the two doublets, the above-mentioned ambiguity does not prevent us from testing the origin of $\mathrm{CP}$ violation, working exclusively within the bosonic sector of the model.

\subsection{Preliminaries}

For spontaneous CP violation one needs, first of all, at least one non-zero imaginary part of the $J_{i}$ invariants, otherwise CP is conserved in the scalar sector. On top of that all $I_{i}$ invariants must vanish, which is just the condition for $\mathrm{CP}$ invariance of the scalar potential. There exist various ways to detect $\mathrm{CP}$ violation originating from the scalar sector experimentally, usually through measurements of certain CP asymmetries, see e.g. [5, 17, 20, 22, 24-34]. In fact, that is the easy part of the task, the one that is much more challenging is to find an experimental and simple method to verify the conditions for CP symmetry of the potential (4.9) and/or (4.10). Let's focus on the condition (4.9). Since the condition is

\footnotetext{
${ }^{4}$ There is a simple analogy to this in special relativity. We may measure both the energy and threemomentum of a particle in the rest frame of an observer even if neither energy nor three-momentum is Lorentz invariant. This is because there exist Lorentz-invariant quantities that in the rest frame of the observer simplify to either the energy or the three-momentum of the particle.
} 
formulated in terms of an invariant, it could be verified experimentally:

$$
-\frac{4 I_{2 Y 2 Z}}{\operatorname{Im} J_{1}}=\left[4 v^{2} \mu^{2} \operatorname{Re} \lambda_{5}-4 \mu^{4}+v^{4}\left(\operatorname{Im} \lambda_{5}\right)^{2}\right]=0
$$

In order to enable experimental verification of the condition for SCPV1, one is tempted to express it through parameters that appear in Feynman rules, e.g. mixing angles. That can be done and the result is the following:

$$
\left[4 v^{2} \mu^{2} \operatorname{Re} \lambda_{5}-4 \mu^{4}+v^{4}\left(\operatorname{Im} \lambda_{5}\right)^{2}\right]=4\left[\Delta^{2}-\mu^{2}\left(M_{1}^{2} R_{13}^{2}+M_{2}^{2} R_{23}^{2}+M_{3}^{2} R_{33}^{2}\right)\right]=0,
$$

where $\Delta$ is defined through

$$
\Delta_{i j k}=\frac{\left(M_{k}^{2}-M_{j}^{2}\right) R_{j 3} R_{k 3}}{\left(v_{1} R_{i 1}-v_{2} R_{i 2}\right)}
$$

such that $\Delta \equiv v \Delta_{123}$.

From equation (6.3), two strategies are apparent:

- It is clear that if we can find ways to measure the three observables $\mu^{2}, \operatorname{Re} \lambda_{5}$ and $\left(\operatorname{Im} \lambda_{5}\right)^{2}$, we are able to test SCPV1. Determining these three observables will most probably require more than three measurements.

- It is also clear that if one could measure $M_{1}, M_{2}, \alpha_{1,2,3}, \tan \beta$ and $\mu^{2}$, then one would be able to test SCPV1. The neutral masses are of course observables, but $\alpha_{1,2,3}$ and $\tan \beta$ are not all observables due to the inability to distinguish the two Higgs doublets as we have already discussed. However, since $\left(\operatorname{Im} \lambda_{5}\right)^{2}=4 \Delta^{2} / v^{4}$, we can conclude that $\Delta^{2}$ is an observable. Furthermore, $R_{i 3}^{2}$ is unchanged under the transformation in eqs. (3.11) and (3.12) of [5] (which amounts to interchanging the two Higgs doublets). Hence $R_{i 3}^{2}$ are observables, and thus can be used in this approach to test SCPV1.

- A third strategy would be to search for a combination of vertex couplings that equals the expression (6.3). Since the absolute value of vertex couplings are observables, this would outline a strategy for disentangling the CP nature of the model. But since the Feynman rules are non-trivial, this approach will most likely represent a considerable algebraic challenge.

Similar comments apply to the SCPV2 case (4.10). The difference is that adopting a similar strategy, even more parameters are needed to decide whether CP is broken spontaneously.

\subsection{Determining the potential}

We know that we cannot uniquely reconstruct the full potential and the VEVs from measurements in the scalar sector only. However, measurements in the non-fermionic sector (i.e., independently of Yukawa couplings) would be sufficient to determine the nature of the $\mathrm{CP}$ violation in the scalar sector. We may thus check the nature of a possible $\mathrm{CP}$ violation via a reconstruction of the parts of the potential that can be measured. This could proceed via measurements of masses and couplings. In principle, the masses could all be determined independently: 
- Measure the neutral masses $M_{1}, M_{2}, M_{3}$ (perhaps best done at a muon collider).

- Measure the charged-Higgs mass $M_{H^{ \pm}}$(single production via $W Z$ fusion, or pair production in $\gamma \gamma$ collisions).

The most natural attempt to determine the remaining parameters in a way that is independent of Yukawa couplings (and therefore not sensitive to a Yukawa-coupling specific version of a $2 \mathrm{HDM}$ ) is through measurements of branching ratios for Higgs bosons decaying into vector bosons:

$$
\operatorname{BR}\left(H_{i} \rightarrow Z Z / W^{+} W^{-}\right) \sim g_{H_{i} Z Z}^{2} \sim g_{H_{i} W^{+} W^{-}}^{2} \sim\left(v_{1} R_{i 1}+v_{2} R_{i 2}\right)^{2}, \quad i=1,2,3
$$

and

$$
\mathrm{BR}\left(H_{i} \rightarrow H^{+} W^{-}\right) \sim g_{H_{i} H^{+} W^{-}}^{2} \sim\left(v R_{i 3}\right)^{2}+\left(v_{2} R_{i 1}-v_{1} R_{i 2}\right)^{2}, \quad i=1,2,3
$$

These 6 quantities are however not independent. For a given $i$, the right-hand sides add to $v^{2}$. Since we consider $v^{2}$ known $(v=246 \mathrm{GeV})$, three relations are thus removed. Furthermore, summing the right-hand sides of (6.6) over $i$, we again get $v^{2}$. Thus, the couplings given by (6.5) and (6.6) provide two independent constraints. However the branching ratios depend on the total decay widths, therefore they are sensitive to Yukawa couplings - the feature that we want to avoid. Therefore, in order to eliminate the total width $\Gamma\left(H_{i}\right)$ one has to consider ratios of branching ratios, so eventually one obtains only one useful constraint.

Here, a comment is in order. It is easy to check that $g_{H_{i} Z Z}^{2}, g_{H_{i} W^{+} W^{-}}^{2}$ and $g_{H_{i} H^{+} W^{-}}^{2}$ are invariant under basis transformations, and thus observable. That implies that only quantities formed from $\alpha_{i}$ and $\tan \beta$ that are invariants could be determined through measurements of branching ratios.

In order to test (6.3) we need three more relations. We could use the following decays (note that there are three equations):

$\operatorname{BR}\left(H_{i} \rightarrow H^{+} H^{-}\right) \sim\left|2 M_{H^{ \pm}}^{2} \frac{v_{1} R_{i 1}+v_{2} R_{i 2}}{v^{2}}-\mu^{2} \frac{v_{1} R_{i 2}+v_{2} R_{i 1}}{v_{1} v_{2}}+M_{i}^{2} \frac{v_{1}^{3} R_{i 2}+v_{2}^{3} R_{i 1}}{v_{1} v_{2} v^{2}}+\Delta \frac{v R_{i 3}}{v_{1} v_{2}}\right|^{2}$

or invoke the trilinear neutral-Higgs couplings. In order to eliminate $\Gamma\left(H_{i}\right)$ one has to consider ratios, for instance one can normalize $\operatorname{BR}\left(H_{i} \rightarrow H^{+} H^{-}\right)$to one of those two independent branching ratios discussed above, see (6.5) and (6.6).

Then, within the considered model, the potential could be constructed (up to an ambiguity irrelevant for the CP properties), and equation (6.3) could be verified.

We have outlined a strategy to determine all the nine independent parameters of the potential. The strategy assumes that three neutral and one charged scalar are observed and their gauge and some cubic couplings could be determined through measurements of appropriate branching ratios. Then, of course, it is possible to verify if CP is broken spontaneously. On the other hand it should be realized that equation (6.3) contains only four invariants, one of which $(v)$ is known, so one could hope that only three measurements 
need to be determined: $\operatorname{Re} \lambda_{5},\left(\operatorname{Im} \lambda_{5}\right)^{2}$ and $\mu^{2}$. This observation triggers the question: what is a minimal set of necessary measurements? For instance, if another scalar particle is discovered, be it $\mathrm{H}_{2}$ or $\mathrm{H}^{+}$, would it be possible to test (6.3) assuming an ideal situation such that all couplings involving known scalar particles could be measured? It turns out that since it is hard to exclude non-linear relationships among couplings, it is highly nontrivial to find such a minimal set of observables that are necessary to test (6.3). It also depends on identifying a selection of measurements that could realistically be performed. In order to find a satisfactory solution, a detailed analysis of all the available couplings is needed and this is beyond the scope of the present study.

\subsection{An ideal observable?}

One could have hoped that it would be possible to find an ideal observable $\mathcal{O}_{\text {CPC }}$ such that

$$
\mathcal{O}_{\mathrm{CPC}} \propto\left[4 v^{2} \mu^{2} \operatorname{Re} \lambda_{5}-4 \mu^{4}+v^{4}\left(\operatorname{Im} \lambda_{5}\right)^{2}\right] .
$$

Unfortunately that seems to be quite difficult.

From the plots that we have presented it is clear that one could, at least in principle, prove experimentally that $\mathrm{CP}$ is violated explicitly. For that one needs to measure $M_{1}$, $M_{2}, M_{H^{ \pm}}, \mu^{2}$. In addition $\alpha_{i}$ and $\tan \beta$ must be known (up to ambiguities). Then if the experimental point is located away (taking into account experimental uncertainties) from the red curves and dots then one can conclude that CP is violated explicitly. However, to prove that $\mathrm{CP}$ violation is spontaneous is, in practice, impossible since one would need to prove that an experimentally allowed point in the parameter space is located exactly either on a red curve or a red dot. Since measurements are always accompanied by some errors, explicit CP violation would always be an option. Of course if an experimental point would lie close to a red curve or a red dot then one could argue that it is more natural to assume that indeed CP is violated spontaneously, since that is connected with increased symmetry of the Lagrangian (the symmetry being, of course, CP itself).

\section{Summary}

We can summarize our findings in the following three points:

1. The strategy we adopted uses $\tan \beta, \alpha_{1,2,3}, \mu^{2}, M_{1,2}^{2}$ and $M_{H^{ \pm}}^{2}$ as input. Then $m_{11}^{2}$, $m_{22}^{2}, m_{12}^{2}$ and $\lambda_{i}$ are determined (also $M_{3}^{2}$ is fixed) adopting the stationarity conditions and relations between diagonal and non-diagonal scalar mass-squared matrices. We choose the input masses $M_{1,2}^{2}$ and positive $M_{3}^{2}$ so $v_{1}=v \cos \beta$ and $v_{2}=v \sin \beta$ is the location of a local minimum. Then we check numerically if the minimum is global, if it is not then it was denoted as cyan in the plots. We have also checked if the vacuum is stable by inspecting positivity of the potential, regions where it is not the case were denoted by pink.

2. Parameters that correspond to SCPV lie on borders of regions for explicit CP violation (ECPV). For those parameters there exist two vacua (related by a CP transformation) of the same depth. For fixed $M_{1}, M_{2}, M_{H^{ \pm}}, \alpha_{1}$ and $\tan \beta$ the SCPV1 
corresponds to a one-dimensional manifold (denoted by red curves) while SCPV2 corresponds to a point (red dot) as it is specified by two conditions. Red lines and red dots are located on borders between regions of ECPV (yellow) and regions where a deeper minimum exists (cyan).

3. Red curves/dots could be approached infinitely close remaining in the region of explicit CP violation. Therefore even if the potential parameters were known (always with some uncertainty) SCPV could be mimicked by ECPV. Of course, if parameters are such that the model is far from the red curves/dots, one can conclude that CP is violated explicitly. Perhaps the simplest (theoretically) method to test SCPV1 would be to measure $\operatorname{Im}\left[\left(m_{12}^{2}\right)^{2} \lambda_{5}^{*}\right]$, if that was non-zero, CP would be broken explicitly. In spite of the twofold ambiguity that unavoidably accompanies measurements that are not sensitive to Yukawa couplings, the conditions for spontaneous CPV, SCPV1 and SCPV2 could be verified experimentally.

It should be stressed that in our analysis, no assumptions were made on the structure of Yukawa couplings.

\section{Acknowledgments}

We are grateful to G. Branco and M. N. Rebelo for valuable discussions, and to the NORDITA Program "Beyond the LHC" for hospitality during the final stage of this work. We thank I. Ivanov for bringing to our attention certain aspects of the geometric approach to the 2HDM and for comments concerning regions of CP conservation. The research of P.O. has been supported by the Research Council of Norway. The work of B.G. is supported in part by the National Science Centre (Poland) as a research project, decision no DEC-2011/01/B/ST2/00438.

\section{A Minimum conditions}

We shall here define some notation related to minimizing the potential with respect to an independent set of variables. If we choose these to be $\Phi_{1}^{\dagger}$ and $\Phi_{2}^{\dagger}$, we get from eq. (2.1)

$$
\begin{aligned}
\frac{\partial V\left(\Phi_{1}, \Phi_{2}\right)}{\partial \Phi_{1}^{\dagger}}= & -\frac{1}{2}\left\{m_{11}^{2} \Phi_{1}+m_{12}^{2} \Phi_{2}\right\} \\
& +\lambda_{1}\left(\Phi_{1}^{\dagger} \Phi_{1}\right) \Phi_{1}+\lambda_{3}\left(\Phi_{2}^{\dagger} \Phi_{2}\right) \Phi_{1}+\lambda_{4}\left(\Phi_{2}^{\dagger} \Phi_{1}\right) \Phi_{2}+\lambda_{5}\left(\Phi_{1}^{\dagger} \Phi_{2}\right) \Phi_{2}=0 \\
\frac{\partial V\left(\Phi_{1}, \Phi_{2}\right)}{\partial \Phi_{2}^{\dagger}}= & -\frac{1}{2}\left\{m_{22}^{2} \Phi_{2}+\left(m_{12}^{2}\right)^{*} \Phi_{1}\right\} \\
& +\lambda_{2}\left(\Phi_{2}^{\dagger} \Phi_{2}\right) \Phi_{2}+\lambda_{3}\left(\Phi_{1}^{\dagger} \Phi_{1}\right) \Phi_{2}+\lambda_{4}\left(\Phi_{1}^{\dagger} \Phi_{2}\right) \Phi_{1}+\left(\lambda_{5}\right)^{*}\left(\Phi_{2}^{\dagger} \Phi_{1}\right) \Phi_{1}=0
\end{aligned}
$$

In the "bar'red" basis (2.11), these equations would in general have additional terms involving $\bar{\lambda}_{6,7}$. In all, we have four conditions, two real parts and two imaginary parts must all vanish. 
The real parts of these equations can be used to solve for $m_{11}^{2}$ and $m_{22}^{2}$ in terms of the $\lambda \mathrm{s}$ and the VEVs. Because of hermiticity, the imaginary parts give just one condition

$$
\operatorname{Im} m_{12}^{2}=v_{1} v_{2} \operatorname{Im} \lambda_{5} .
$$

\section{A.1 Stationary-point equations for complex vacuum}

In the case where we have a charge-conserving minimum of the form

$$
\begin{aligned}
& \left\langle\Phi_{1}\right\rangle=\frac{1}{\sqrt{2}}\left(\begin{array}{c}
0 \\
v_{1}
\end{array}\right) \\
& \left\langle\Phi_{2}\right\rangle=\frac{1}{\sqrt{2}}\left(\begin{array}{c}
0 \\
v_{2} e^{i \theta}
\end{array}\right)
\end{aligned}
$$

the stationary-point equations are:

$$
\begin{aligned}
\lambda_{1} v_{1}^{3}+\left(\lambda_{3}+\lambda_{4}+\operatorname{Re} \lambda_{5} \cos 2 \theta-\operatorname{Im} \lambda_{5} \sin 2 \theta\right) v_{1} v_{2}^{2} & -m_{11}^{2} v_{1}-\left(\operatorname{Re}\left(m_{12}^{2}\right) \cos \theta-\operatorname{Im}\left(m_{12}^{2}\right) \sin \theta\right) v_{2} \\
= & 0 \\
\lambda_{2} \cos \theta v_{2}^{3}+\left[\left(\lambda_{3}+\lambda_{4}+\operatorname{Re} \lambda_{5}\right) \cos \theta-\operatorname{Im}\left(\lambda_{5}\right) \sin \theta\right] v_{1}^{2} v_{2} & \\
-\operatorname{Re}\left(m_{12}^{2}\right) v_{1}-m_{22}^{2} \cos \theta v_{2} & =0 \\
\left(\operatorname{Im} \lambda_{5} \cos 2 \theta+\operatorname{Re} \lambda_{5} \sin 2 \theta\right) v_{1} v_{2}^{2}-\left(\operatorname{Im}\left(m_{12}^{2}\right) \cos \theta+\operatorname{Re}\left(m_{12}^{2}\right) \sin \theta\right) v_{2} & =0 \\
\lambda_{2} \sin \theta v_{2}^{3}+\left[\left(\lambda_{3}+\lambda_{4}-\operatorname{Re} \lambda_{5}\right) \sin \theta-\operatorname{Im}\left(\lambda_{5}\right) \cos \theta\right] v_{1}^{2} v_{2} & \\
+\operatorname{Im}\left(m_{12}^{2}\right) v_{1}-m_{22}^{2} \sin \theta v_{2} & =0
\end{aligned}
$$

We note that these are necessary, but not sufficient, conditions for having a minimum of the potential. It is also worth noticing that for $\theta=0$ equations (A.6) and (A.7) coincide.

It is also instructive to write the stationary-point conditions as two complex equations:

$$
\begin{aligned}
& \lambda_{1} v_{1}^{3}+\left[\lambda_{3}+\lambda_{4}+\left|\lambda_{5}\right| e^{i(\gamma+2 \theta)}\right] v_{1} v_{2}^{2}-m_{11}^{2} v_{1}-\left|m_{12}^{2}\right| e^{i(\alpha+\theta)} v_{2}=0 \\
& \lambda_{2} v_{2}^{3}+\left[\lambda_{3}+\lambda_{4}+\left|\lambda_{5}\right| e^{i(\gamma+2 \theta)}\right] v_{1}^{2} v_{2}-m_{22}^{2} v_{2}-\left|m_{12}^{2}\right| e^{i(\alpha+\theta)} v_{1}=0
\end{aligned}
$$

\section{B Observable parameters of the potential}

In this appendix we will show how different parameters of the potential (and combinations thereof) can be written in an invariant form that is independent of our choice of basis. If the parameters can be written in an invariant form, it means that they are observables and can be measured. Let us start by writing the potential and the VEVs in the forms $[8,9]$

$$
V=Y_{a \bar{b}} \Phi_{\bar{a}}^{\dagger} \Phi_{b}+\frac{1}{2} Z_{a \bar{b} c \bar{d}}\left(\Phi_{\bar{a}}^{\dagger} \Phi_{b}\right)\left(\Phi_{\bar{c}}^{\dagger} \Phi_{d}\right),
$$

and

$$
\left\langle\Phi_{a}\right\rangle=\frac{1}{\sqrt{2}}\left(\begin{array}{c}
0 \\
v \hat{v}_{a}
\end{array}\right) .
$$


Comparing this to (2.1) and (2.2), we find that

$$
\begin{aligned}
\hat{v}_{1}=\frac{v_{1}}{v}, & \hat{v}_{2}=\frac{v_{2}}{v}, \\
Y_{11}=-\frac{m_{11}^{2}}{2}, & Y_{12}=-\frac{m_{12}^{2}}{2}, \quad Y_{21}=-\frac{\left(m_{12}^{2}\right)^{*}}{2}, \quad Y_{22}=-\frac{m_{22}^{2}}{2}
\end{aligned}
$$

and

$$
\begin{aligned}
& Z_{1111}=\lambda_{1}, \quad Z_{2222}=\lambda_{2}, \\
& Z_{1122}=Z_{2211}=\lambda_{3}, \\
& Z_{1221}=Z_{2112}=\lambda_{4}, \\
& Z_{1212}=\lambda_{5}, \quad Z_{2121}=\left(\lambda_{5}\right)^{*} .
\end{aligned}
$$

All other $Z_{a \bar{b} c \bar{d}}$ vanish. In [8,9] it is shown how to construct basis-invariant quantities from contractions between tensor indices of $V_{a \bar{b}}, Y_{a \bar{b}}$ and $Z_{a \bar{b}} \bar{d} \bar{d}$ following a certain pattern. Using the same pattern, we are able to write parameters of our potential in an invariant way. Every quantity where we construct a scalar by contracting barred against unbarred indices in the $V$-, $Y$ - and $Z$-tensors will be a basis-invariant. Let us first define the following matrices:

$$
\begin{aligned}
& V_{a \bar{b}}=\hat{v}_{a} \hat{v}_{\bar{b}}^{*}=\frac{1}{v^{2}}\left(\begin{array}{cc}
v_{1}^{2} & v_{1} v_{2} \\
v_{1} v_{2} & v_{2}^{2}
\end{array}\right), \\
& Z_{a \bar{b}}^{(1)}=Z_{a \bar{c} c \bar{b}}=\left(\begin{array}{cc}
\lambda_{1}+\lambda_{4} & 0 \\
0 & \lambda_{2}+\lambda_{4}
\end{array}\right), \quad Z_{a \bar{b}}^{(2)}=Z_{a \bar{b} c \bar{c}}=\left(\begin{array}{cc}
\lambda_{1}+\lambda_{3} & 0 \\
0 & \lambda_{2}+\lambda_{3}
\end{array}\right) . \\
& Z_{c \bar{d}}^{(21)}=Z_{a \bar{b}}^{(2)} Z_{b \bar{a} c \bar{d}}, \quad \quad Z_{c \bar{d}}^{(V)}=V_{a \bar{b}} Z_{b \bar{a} c \bar{d}}
\end{aligned}
$$

Consider the invariant expressions

$$
\begin{aligned}
& \frac{1}{2}\left[\operatorname{Tr} Z^{(2)}-\frac{\operatorname{Tr}\left(Z^{(2)}\right)^{2}-2 \operatorname{Tr}\left(V Z^{(21)}\right)}{\operatorname{Tr} Z^{(2)}-2 \operatorname{Tr}\left(V Z^{(2)}\right)}\right]=\lambda_{3} \\
& \frac{1}{2}\left[\operatorname{Tr} Z^{(1)}-\frac{\operatorname{Tr}\left(Z^{(2)}\right)^{2}-2 \operatorname{Tr}\left(V Z^{(21)}\right)}{\operatorname{Tr} Z^{(2)}-2 \operatorname{Tr}\left(V Z^{(2)}\right)}\right]=\lambda_{4} .
\end{aligned}
$$

Since these clearly invariant expressions simplify to $\lambda_{3}$ and $\lambda_{4}$ in our model, $\lambda_{3}$ and $\lambda_{4}$ are observables in our model.

The parameters $\lambda_{1}$ and $\lambda_{2}$, however, are not observables. This is due to the fact that the labeling of the two doublets $\Phi_{1}$ and $\Phi_{2}$ is arbitrary, and interchanging the two doublets will just amount to renaming the parameters of the potential. This symmetry of the potential is written out explicitly in eqs. (3.11) and (3.12) of [5]. Therefore we will not be able to measure parameters that would let us distinguish one doublet from the other by performing measurements in the scalar sector only. Hence, parameters like $\lambda_{1}, \lambda_{2}$ and $\tan \beta$ cannot be determined uniquely, unless one specifies the Yukawa couplings. In other words, certain combinations of parameters that are symmetric under the interchange of the two doublets are observables:

$$
\begin{aligned}
\operatorname{Tr} Z^{(2)}-2 \lambda_{3} & =\lambda_{1}+\lambda_{2} \\
2 \operatorname{Tr}\left(Z^{(2)}\right)^{2}-\left(\operatorname{Tr} Z^{(2)}\right)^{2} & =\left(\lambda_{1}-\lambda_{2}\right)^{2}
\end{aligned}
$$


Here, the fact that $\lambda_{3}$ has been shown to be an observable leads to the conclusion that $\lambda_{1}+\lambda_{2}$ is an observable. Together with the observable $\left(\lambda_{1}-\lambda_{2}\right)^{2}$ this means that one is able to determine the values of $\lambda_{1}$ and $\lambda_{2}$, but one is not able to determine which is which, i.e., there is a twofold ambiguity in the determination of these two parameters.

The same goes for $\tan \beta$ (or equivalently $v_{1}$ and $v_{2}$ ). The quantity $v_{1}^{2}+v_{2}^{2}=v^{2}=$ $(246 \mathrm{GeV})^{2}$ is invariant under a change of basis. Also consider

$$
\frac{v^{4}\left(\operatorname{Tr} Z^{(2)}-2 \operatorname{Tr}\left(V Z^{(2)}\right)\right)^{2}}{2 \operatorname{Tr}\left(Z^{(2)}\right)^{2}-\left(\operatorname{Tr} Z^{(2)}\right)^{2}}=\left(v_{1}^{2}-v_{2}^{2}\right)^{2}
$$

The fact that $v_{1}^{2}+v_{2}^{2}$ and $\left(v_{1}^{2}-v_{2}^{2}\right)^{2}$ are observables (together with the fact that $v_{i}$ is positive) means that $v_{1}$ and $v_{2}$ can be determined up to the twofold ambiguity.

We find invariant expressions also for $\operatorname{Re} \lambda_{5}$ and $\mu^{2}$. Thus, these two parameters are also observables in our model. We have not substituted the invariant expressions for $\left(\lambda_{1}-\lambda_{2}\right)^{2}$ or $\left(v_{1}^{2}-v_{2}^{2}\right)^{2}$ in the following expressions. For $\operatorname{Re} \lambda_{5}$, the expression is

$$
\begin{aligned}
& \frac{v^{4}}{\left(\lambda_{1}-\lambda_{2}\right)^{2}\left[\left(v_{1}^{2}-v_{2}^{2}\right)^{2}-v^{4}\right]}\left[-2 \operatorname{Tr}\left(V Z^{(V)}\right)\left(\lambda_{1}-\lambda_{2}\right)^{2}-\operatorname{Tr}\left(Z^{(2)} Z^{(21)}\right)\right. \\
& +3 \operatorname{Tr}\left(V Z^{(21)}\right)\left(2 \operatorname{Tr}\left(V Z^{(2)}\right)-\operatorname{Tr} Z^{(2)}\right)+\operatorname{Tr}\left(V Z^{(2)}\right)\left(\operatorname{Tr}\left(Z^{(2)}\right)^{2}+2 \operatorname{Tr} Z^{(2)} \operatorname{Tr} Z^{(1)}\right) \\
& \left.-2\left(\operatorname{Tr}\left(V Z^{(2)}\right)\right)^{2}\left(\operatorname{Tr} Z^{(2)}+\operatorname{Tr} Z^{(1)}\right)+\operatorname{Tr}\left(Z^{(2)}\right)^{2}\left(\operatorname{Tr} Z^{(2)}+\operatorname{Tr} Z^{(1)}\right)-\left(\operatorname{Tr} Z^{(2)}\right)^{2} \operatorname{Tr} Z^{(1)}\right] \\
& =\operatorname{Re} \lambda_{5},
\end{aligned}
$$

and for $\mu^{2}$

$$
\begin{aligned}
& \frac{v^{2}}{\left(\lambda_{1}-\lambda_{2}\right)^{2}\left[\left(v_{1}^{2}-v_{2}^{2}\right)^{2}-v^{4}\right]}\left[-2 \operatorname{Tr} Y\left(\operatorname{Tr}\left(V Z^{(21)}\right)-\operatorname{Tr}\left(V Z^{(2)}\right) \operatorname{Tr} Z^{(2)}\right)\right. \\
& -2 \operatorname{Tr}\left(Y Z^{(2)}\right)\left(2 \operatorname{Tr}\left(V Z^{(2)}\right)-\operatorname{Tr} Z^{(2)}\right)+v^{2} \operatorname{Tr}\left(V Z^{(V)}\right)\left(\left(\operatorname{Tr} Z^{(2)}\right)^{2}-2 \operatorname{Tr}\left(Z^{(2)}\right)^{2}\right) \\
& \left.-v^{2} \operatorname{Tr}\left(V Z^{(2)}\right)\left(\operatorname{Tr}\left(V Z^{(21)}\right)-\operatorname{Tr}\left(Z^{(2)}\right)^{2}\right)-2 \operatorname{Tr}\left(Y Z^{(21)}\right)-v^{2} \operatorname{Tr}\left(Z^{(V)} Z^{(21)}\right)\right] \\
& =\mu^{2}
\end{aligned}
$$

Finally, we consider $\operatorname{Im} \lambda_{5}$, which we can only determine up to a sign ambiguity because of the inability to distinguish the two doublets. Consider

$$
\frac{4 \mu^{4}-4 v^{2} \mu^{2} \operatorname{Re} \lambda_{5}-4 I_{2 Y 2 Z} / \operatorname{Im} J_{1}}{v^{4}}=\left(\operatorname{Im} \lambda_{5}\right)^{2} .
$$

Since we have already shown that $\operatorname{Re} \lambda_{5}$ and $\mu^{2}$ are observables, it follows that $\left(\operatorname{Im} \lambda_{5}\right)^{2}$ is an observable.

In summary then, all parameters of the potential and the VEVs can in principle be measured without specifying the Yukawa sector, up to the ambiguities: (i) $\lambda_{1} \leftrightarrow \lambda_{2}$, (ii) $\operatorname{Im} \lambda_{5} \leftrightarrow-\operatorname{Im} \lambda_{5}$ and (iii) $v_{1} \leftrightarrow v_{2}$. These ambiguities are not independent. If one of them is resolved (meaning that we have been able to distinguish between the two doublets), the two others will resolve simultaneously. 
Open Access. This article is distributed under the terms of the Creative Commons Attribution License (CC-BY 4.0), which permits any use, distribution and reproduction in any medium, provided the original author(s) and source are credited.

\section{References}

[1] G. Branco et al., Theory and phenomenology of two-Higgs-doublet models, Phys. Rept. 516 (2012) 1 [arXiv: 1106.0034] [INSPIRE].

[2] L. Lavoura, Signatures of discrete symmetries in the scalar sector, Phys. Rev. D 50 (1994) 7089 [hep-ph/9405307] [INSPIRE].

[3] I.P. Ivanov, Minkowski space structure of the Higgs potential in 2HDM. II. Minima, symmetries and topology, Phys. Rev. D 77 (2008) 015017 [arXiv:0710.3490] [INSPIRE].

[4] E. Accomando et al., Workshop on CP Studies and Non-Standard Higgs Physics, hep-ph/0608079 [INSPIRE].

[5] A.W. El Kaffas, W. Khater, O.M. Ogreid and P. Osland, Consistency of the two Higgs doublet model and CP-violation in top production at the LHC, Nucl. Phys. B 775 (2007) 45 [hep-ph/0605142] [INSPIRE].

[6] I. Ivanov, Minkowski space structure of the Higgs potential in 2HDM, Phys. Rev. D 75 (2007) 035001 [Erratum ibid. D 76 (2007) 039902] [hep-ph/0609018] [INSPIRE].

[7] A. Wahab El Kaffas, P. Osland and O.M. Ogreid, Constraining the Two-Higgs-Doublet-Model parameter space, Phys. Rev. D 76 (2007) 095001 [arXiv:0706.2997] [INSPIRE].

[8] S. Davidson and H.E. Haber, Basis-independent methods for the two-Higgs-doublet model, Phys. Rev. D 72 (2005) 035004 [Erratum ibid. D 72 (2005) 099902] [hep-ph/0504050] [INSPIRE].

[9] J.F. Gunion and H.E. Haber, Conditions for CP-violation in the general two-Higgs-doublet model, Phys. Rev. D 72 (2005) 095002 [hep-ph/0506227] [INSPIRE].

[10] I. Ivanov, Two-Higgs-doublet model from the group-theoretic perspective, Phys. Lett. B 632 (2006) 360 [hep-ph/0507132] [inSPIRE].

[11] M. Maniatis, A. von Manteuffel and O. Nachtmann, CP violation in the general two-Higgs-doublet model: A Geometric view, Eur. Phys. J. C 57 (2008) 719 [arXiv:0707.3344] [INSPIRE].

[12] C.C. Nishi, CP violation conditions in N-Higgs-doublet potentials, Phys. Rev. D 74 (2006) 036003 [Erratum ibid. D 76 (2007) 119901] [hep-ph/0605153] [INSPIRE].

[13] S.L. Glashow and S. Weinberg, Natural Conservation Laws for Neutral Currents, Phys. Rev. D 15 (1977) 1958 [INSPIRE].

[14] B. Grzadkowski, O. Ogreid and P. Osland, Natural Multi-Higgs Model with Dark Matter and CP-violation, Phys. Rev. D 80 (2009) 055013 [arXiv:0904.2173] [INSPIRE].

[15] L. Lavoura and J.P. Silva, Fundamental CP-violating quantities in a $\mathrm{SU}(2) \times \mathrm{U}(1)$ model with many Higgs doublets, Phys. Rev. D 50 (1994) 4619 [hep-ph/9404276] [INSPIRE].

[16] F. Botella and J.P. Silva, Jarlskog-like invariants for theories with scalars and fermions, Phys. Rev. D 51 (1995) 3870 [hep-ph/9411288] [INSPIRE]. 
[17] W. Khater and P. Osland, CP violation in top quark production at the LHC and two Higgs doublet models, Nucl. Phys. B 661 (2003) 209 [hep-ph/0302004] [InSPIRE].

[18] A.W. El Kaffas, P. Osland and O.M. Ogreid, CP violation, stability and unitarity of the two Higgs doublet model, Nonlin. Phenom. Complex Syst. 10 (2007) 347 [hep-ph/0702097] [INSPIRE].

[19] S. Kanemura, T. Kubota and E. Takasugi, Lee-Quigg-Thacker bounds for Higgs boson masses in a two doublet model, Phys. Lett. B 313 (1993) 155 [hep-ph/9303263] [INSPIRE].

[20] L. Basso et al., Probing the charged Higgs boson at the LHC in the CP-violating type-II 2HDM, JHEP 11 (2012) 011 [arXiv:1205.6569] [INSPIRE].

[21] L. Basso et al., The CP-violating type-II 2HDM and Charged Higgs boson benchmarks, PoS (Corfu2012) 029 [arXiv: 1305.3219] [INSPIRE].

[22] B. Grzadkowski and J. Pliszka, Testing scalar sector CP-violation in top quark production and decay at linear $e^{+} e^{-}$colliders, Phys. Rev. D 63 (2001) 115010 [hep-ph/0012110] [INSPIRE].

[23] B. Grzadkowski, J.F. Gunion and J. Pliszka, How valuable is polarization at a muon collider? A Test case: Determining the CP nature of a Higgs boson, Nucl. Phys. B 583 (2000) 49 [hep-ph/0003091] [INSPIRE].

[24] B. Grzadkowski, J.F. Gunion and J. Kalinowski, Finding the CP-violating Higgs bosons at $e^{+} e^{-}$colliders, Phys. Rev. D 60 (1999) 075011 [hep-ph/9902308] [INSPIRE].

[25] B. Grzadkowski and Z. Hioki, CP violating lepton energy correlation in $e^{-} e^{+} \rightarrow t \bar{t}$, Phys. Lett. B 391 (1997) 172 [hep-ph/9608306] [INSPIRE].

[26] J.F. Gunion, B. Grzadkowski and X.-G. He, Determining the top - anti-top and ZZ couplings of a neutral Higgs boson of arbitrary CP nature at the NLC, Phys. Rev. Lett. 77 (1996) 5172 [hep-ph/9605326] [INSPIRE].

[27] B. Grzadkowski, CP violation in $H \rightarrow t \bar{t}$ decays at $e^{+} e^{-}$colliders, Phys. Lett. B 338 (1994) 71 [hep-ph/9404330] [INSPIRE].

[28] D. Chang and W.-Y. Keung, CP violation in the decay of neutral Higgs boson into $t-\bar{t}$ and $W^{+}-W^{-}$, Phys. Lett. B 305 (1993) 261 [hep-ph/9301265] [INSPIRE].

[29] G.-H. Wu and A. Soni, Novel CP-violating effects in B decays from charged Higgs in a two Higgs doublet model for the top quark, Phys. Rev. D 62 (2000) 056005 [hep-ph/9911419] [INSPIRE].

[30] S. Bar-Shalom, D. Atwood and A. Soni, Two Higgs doublets models and CP-violating Higgs exchange in $e^{+} e^{-} \rightarrow t \bar{t} Z$, Phys. Lett. B 419 (1998) 340 [hep-ph/9707284] [InSPIRE].

[31] S. Bar-Shalom, D. Atwood, G. Eilam, R. Mendel and A. Soni, Large tree level CP-violation in $e^{+} e^{-} \rightarrow t \bar{t} H^{0}$ in the two Higgs doublet model, Phys. Rev. D 53 (1996) 1162 [hep-ph/9508314] [INSPIRE].

[32] D. Atwood and A. Soni, Neutral Higgs CP-violation at $\mu^{+} \mu^{-}$colliders, Phys. Rev. D 52 (1995) 6271 [hep-ph/9505233] [INSPIRE].

[33] A. Soni and R. Xu, Probing CP-violation via Higgs decays to four leptons, Phys. Rev. D 48 (1993) 5259 [hep-ph/9301225] [INSPIRE].

[34] A. Skjold and P. Osland, Signals of CP-violation in Higgs decay, Phys. Lett. B 329 (1994) 305 [hep-ph/9402358] [INSPIRE]. 